\title{
NOON states via a quantum walk of bound particles
}

\author{
Enrico Compagno, ${ }^{1}$ Leonardo Banchi, ${ }^{1}$ Christian Gross, ${ }^{2}$ and Sougato Bose ${ }^{1}$ \\ ${ }^{1}$ Department of Physics and Astronomy, University College London, Gower Street, WC1E 6BT London, United Kingdom \\ ${ }^{2}$ Max-Planck-Institut für Quantenoptik, 85748 Garching, Germany
}

(Received 17 October 2016; published 9 January 2017)

\begin{abstract}
Tight-binding lattice models allow the creation of bound composite objects which, in the strong-interacting regime, are protected against dissociation. We show that a local impurity in the lattice potential can generate a coherent split of an incoming bound particle wave packet which consequently produces a NOON state between the endpoints. This is nontrivial because, when finite lattices are involved, edge-localization effects render challenging their use for nonclassical state generation and information transfer. We derive an effective model to describe the propagation of bound particles in a Bose-Hubbard chain. We introduce local impurities in the lattice potential to inhibit localization effects and to split the propagating bound particle, thus enabling the generation of distant NOON states. We analyze how minimal engineering transfer schemes improve the transfer fidelity and we quantify the robustness to typical decoherence effects in optical lattice implementations. Our scheme potentially has an impact on quantum-enhanced atomic interferometry in a lattice.
\end{abstract}

DOI: 10.1103/PhysRevA.95.012307

\section{INTRODUCTION}

The unprecedented ability to control and observe multiparticle states in optical lattice systems with single-site resolution [1-14] makes possible the investigation of new quantum interference effects. Indeed, the dynamics of quantum interacting systems display many interesting features that go beyond the regime traditionally studied in linear optics. From the fundamental perspective it is then important to understand how to exploit the natural interactions to "engineer" the many-particle dynamics in a lattice for creating nonclassical states, such as multiparticle NOON states. Compared with classical setups, and also to other schemes for atom interferometry $[15,16]$, the advantage of this approach is that nonclassical states (e.g., NOON and dual Fock states [17]) enhance the estimation precision of the phase difference between the output arms of an interferometer [18-21], making them highly attractive for technological applications. Super-resolution for NOON states with $N=2,3$ has been recently shown experimentally for microscopy purposes [22]. However, the generation of nonclassical states with high fidelity is still a hard task. For instance, in existing photonic realizations, NOON states with $N=5$ have been demonstrated, but with a limited $42 \%$ fringe visibility [23-25]. Moreover, with those schemes, there is a theoretical upper threshold for the state preparation fidelity of $94.3 \%$ [23]. It is therefore important to develop alternative schemes for high-fidelity NOON-state generation.

To sense spatial inhomogeneities and to probe external fields localized over few sites, it is convenient for the components of the NOON state to be spatially well separated. In this context a quantum walk of interacting atoms in an optical lattice might be very useful, as we shall explore. For a lattice setup, this type of scheme is important because it enables one to avoid the necessity of measurement based schemes [26,27] (which are still challenging in current optical lattice experiments with few particles), time-dependent external potentials [28], engineered-bath-based schemes [29], or ring lattices [30,31]. From the theoretical point of view, optical lattice systems in a low filling limit are modeled by the Bose-Hubbard Hamiltonian, which contains a hopping term between neighboring sites and an onsite interaction. If more than one particle is initially located in the same site and the onsite interaction is sufficiently strong, this model favors the creation of bound states, which are stable against dissociation [2,32-36]. A natural question is then whether bound states have some advantages for nonclassical state production tasks. The key point here is that a bound state behaves like an effective single particle for strong-enough interaction and together with a balanced beam splitter it can be used to produce a high-fidelity NOON state. When distant sites are involved, a balanced beam splitter transformation can be obtained via the particle dynamics by introducing suitable impurities in the lattice potential. These impurities generate a coherent splitting of the wave packet of propagating particles which enables high-efficiency effective linear optical operations between remote sites of finite lattices [37,38]. Even without the onsite interaction, peculiar quantum interference effects enable the production of nonclassical states; namely, two-particle NOON states, via the celebrated Hong-Ou-Mandel effect [37,38]. Being a linear-optical effect, the efficiency of this protocol is maximized when the atom-atom interaction is kept in the weak-coupling regime.

On the other hand it is intriguing to investigate the strongly interacting regime; namely, whether the role of the interatomic interaction can be exploited as a resource to generate a wider class of nonclassical states; for instance, high NOON states $(N>2)$ between distant sites. However, as far as distant sites are concerned, the realizability of this scheme is hindered by the possibility to engender and control the tunneling dynamics of a bound state initially located in one edge of the lattice. The main obstacle is the presence of edge-locked states which inhibit the hopping dynamics $[39,40]$. Edge-locking indeed creates an effective energy barrier between edge and bulk sites, which suppresses the bound-state propagation along the lattice.

In other words, it is still an open problem how to tune the lattice potential to realize transformations between far sites when strongly interacting particles are involved. Recently, in the case of fermions, a long-range state transfer protocol for a two-particle bound state has been studied in a one- and two-dimensional lattices by using ac fields. In this scheme 


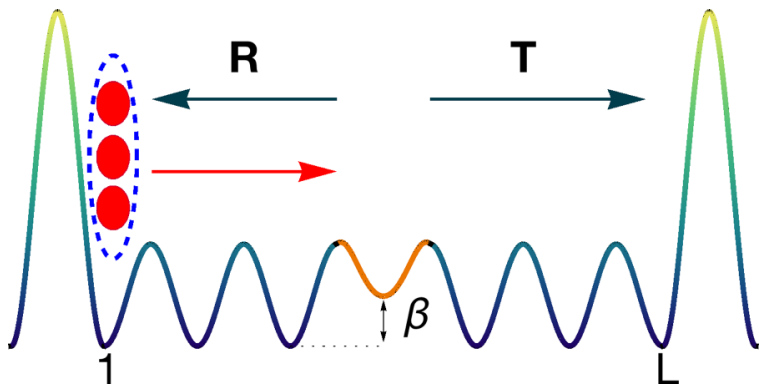

FIG. 1. Scheme of the model: when a bound state is initially in the site 1 of a finite lattice, a suitably introduced local impurity (orange) in the chemical potential $\mu_{j}=-\beta \delta_{j, L / 2+1}$ triggers a wavepacket splitting in a reflected $R$ and a transmitted $T$ component. If appropriately tuned it generates a NOON state between the endpoints (site 1 and $L$ ) at the transfer time. The two green peaks are local fields which realize a finite lattice model. The red arrow represents the natural direction of propagation of the bound particle once lowered the lattice depth at time $t=0$. We also add local fields in the first and last sites of the chain to inhibit the edge-localization effect and then to delocalize the bound particle from the edges.

the state transfer takes place only between edge states, while bulk sites remain empty during the dynamics of the system $[41,42]$. On the other hand, for bosonic particles, the underbarrier tunneling of a dimer has been analyzed in Refs. [43,44]. Nonetheless, the problem of how to transfer bound states with high fidelity over arbitrarily long distances in engineered finitesize chains has not been completely addressed yet, in particular when more than two particle bound states are involved in the dynamics.

In this paper we analyze the bound particle dynamics in a finite lattice by mapping the Bose-Hubbard Hamiltonian into an effective single-particle chain via a strong-coupling expansion in the onsite interaction term (see Fig. 1). This mapping is realized by applying the effective theory developed in Ref. [45]. We study the conditions to prevent the dissociation of the bound particle during the dynamics and we show that, when these conditions are satisfied, the bound particle evolution is perfectly described by our effective model. We find the connection between the effective hopping rates, which are interaction dependent, and the physical parameters of the of the Bose-Hubbard model. We then show how to design these parameters such that the effective evolution produces a splitting transformation suitable for creating NOON states between distant sites. Applications in quantum enhanced metrology are thus discussed.

The first step towards the realization of our protocol is to develop a method to delocalize a bound-state wave packet from the edges of a finite chain. In the spin-chain case the edge-locking effect for spin blocks is bypassed using $\pi$ pulses to flip the leftmost spin and then enable the wave-packet delocalization $[40,46]$. On the other hand, in the Bose-Hubbard model an operative method to unlock bound particle states has not been proposed yet. Here we show that the edge-locking effect can be eliminated by introducing static impurities in the chemical potential localized at the endpoints. These impurities, which can be generated by using external local fields, compensates the energy gap between edge and bulk sites and enable the dynamics. After "unlocking" the dynamics, we study the state transfer efficiency for a uniform chain and the robustness from typical environmental effects; specifically decoherence effects due to spontaneous emission in an optical lattice setup. Moreover we show how a minimal engineering of the hopping rates can enhance the transfer efficiency, specifically tuning the first and the last tunneling couplings of the chain. We show then how to add an extra impurity in the middle of the chain to generate a NOON state between the edges of the lattice. We derive analytical expressions for the optimal parameters to generate $N=2$ and $N=3$ NOON states, and we show how our approach can be straightforwardly generalized for producing larger "cat" states. Finally, we show how to experimentally detect the generated NOON state by using the technology available nowadays.

\section{MAIN IDEA}

We consider a one-dimensional chain of length $L$, described by a Bose-Hubbard model with site dependent parameters according to the following Hamiltonian [47]:

$$
\begin{aligned}
H= & -\sum_{j=1}^{L-1} \frac{J_{j}}{2}\left[a_{j} a_{j+1}^{\dagger}+\text { H.c. }\right] \\
& +\sum_{j=1}^{L} \frac{U_{j}}{2} n_{j}\left(n_{j}+1\right)-\sum_{j=1}^{L} \mu_{j} n_{j} .
\end{aligned}
$$

Here $a_{j}\left(a_{j}^{\dagger}\right)$ are the boson annihilation (creation) operators, $n_{j}=a_{j}^{\dagger} a_{j}$ are the number operators and $J_{j}, U_{j}$, and $\mu_{j}$ are respectively the hopping rate, the onsite interaction, and the chemical potential. Because the Hamiltonian (1) preserves the total number of excitations of the system, the dynamics can be evaluated in a Hilbert subspace with a fixed number of particles.

One characteristic feature of the Bose-Hubbard model is that the onsite interaction enables the creation of "bound" states when several particles are in the same site [2,32-36]. Here we are interested in a nonequilibrium configuration, in the low-filling regime, where $M$ particles are initially located on a single site. In optical lattices the initialization of the system in one of these states is obtained starting from the Mott-Insulator regime and using single-atom addressing techniques [1-4,6,11]. The key point here is that, provided that the onsite interaction strength $U$ is large enough, the resulting state composed of $M>1$ bounded particles on the same site is stable against dissociation during the time evolution [32,33,35,36,48,49] and behaves like an effective single particle. Indeed, as explicitly discussed in Refs. [35,36] for a few values of $M$, the bounded-particle states lie in an energy band which is well separated (by an energy separation $\propto U)$ from other states, provided that $U$ is suitably large. In the following we introduce a general theory to model the effective interactions between stable bounded particles.

In general when $U_{j} \gg J_{j}, \mu_{j}$ the different Hilbert subspaces $\mathcal{H}_{M}$ spanned by the states with $M$ bounded particles; namely, $\mathcal{H}_{M}=\left\{|\{M\}, j\rangle=\left(a_{j}^{\dagger}\right)^{M}|0\rangle / \sqrt{M !}: j=1, \ldots, L\right\}$, are energetically well separated. Because of this energy separation between subspaces, if the initial state is composed of a 
bound $M$-particle states, then with a good approximation the dynamics remains confined inside $\mathcal{H}_{M}$. The resulting effective dynamics can be described with a Hamiltonian $H_{M}^{\text {eff }}$ which describes the effective interactions inside $\mathcal{H}_{M}$. By exploiting the theory presented in Ref. [45], which assumes that the dynamical effective subspace is energetically separated by the rest of the Hilbert space, we find that Eq. (1) generates in $\mathcal{H}_{M}$ the effective interaction

$$
H_{M}^{\text {eff }}=\left(\begin{array}{ccccc}
B_{1}^{\text {eff }} & J_{1}^{\text {eff }} & & & \\
J_{1}^{\text {eff }} & B_{2}^{\text {eff }} & J_{2}^{\text {eff }} & & \\
& \ddots & \ddots & \ddots & \\
& & J_{L-2}^{\text {eff }} & B_{L^{-}}^{\text {eff }} & J_{L-1}^{\text {eff }} \\
& & & J_{L-1}^{\text {eff }} & B_{L}^{\text {eff }}
\end{array}\right)
$$

in the basis $|\{M\}, j\rangle, j=1, \ldots, L$. The above Hamiltonian describes a quantum walk of a one-dimensional bounded particle. We mention that, recently, by using a different approach, an effective spin-chain model has been obtained to control and manipulate a one-dimensional (1D) strongly interacting two-specie Bose-Hubbard for quantum communication and computation purposes [50]. Quantum walks have been subject to intensive investigations over the past years, both with single particles and multiparticles [2,48,49,51-54]. In particular, different schemes have been found to engineer the couplings $J_{j}^{\text {eff }}$ and the energies $B_{j}^{\text {eff }}$ such that the dynamics either produces a perfect state transfer [55-57] or a perfect splitting and reconstruction of the initial wave packet; namely, a fractional revival $[38,58,59]$. From our perspective, a perfect state transfer in the effective subspace would give rise to a perfect transmission of a bounded particle; namely, the state $|\{M\}, 1\rangle \propto\left(a_{1}^{\dagger}\right)^{M}|0\rangle$ is dynamically transferred to the opposite end of the chain $|\{M\}, L\rangle \propto\left(a_{L}^{\dagger}\right)^{M}|0\rangle$. Another important application is the perfect fractional revival, which effectively generates a beam-splitting transformation between the ends of the chain. The main reason for its importance is that, when bound states are involved in the perfect splitting transformation $|\{M\}, 1\rangle \rightarrow|\{M\}, 1\rangle+e^{i \phi}|\{M\}, L\rangle$, then an $M$-particle NOON state $\left[\left(a_{1}^{\dagger}\right)^{M}+e^{i \phi}\left(a_{L}^{\dagger}\right)^{M}\right]|0\rangle$ is produced.

The main idea of our scheme is then to engineer the couplings $J_{j}$ and the chemical potentials $\mu_{j}$ in the BoseHubbard (1), such that the effective couplings in Eq. (2) have the suitable pattern for either state transfer or state splitting (fractional revival). The timescale of the resulting effective dynamical transformation is approximately given by $1 / J_{\text {eff }}$. However, since the effective hopping in $\mathcal{H}_{M}$ involves $M-1$ "virtual" transitions through states which are outside $\mathcal{H}_{M}$, then it is simple to realize that $J_{j}^{\text {eff }} \propto J_{j}^{M} / U_{j}^{M-1}$, so $J_{j}^{\text {eff }}$ exponentially decreases with $M$ for large $U$. For larger $M$-particle bound states the effective evolution thus become slow and the efficiency of the scheme may be severely affected by environmental effects. Because of this, in the next sections we thoroughly analyze the $M=2$ and $M=3$ cases which are more feasible, given the current experimental capabilities. The overall theoretical scheme is however fully general and can be readily extended for larger values of $M$.

\section{APPLICATIONS}

\section{A. Edge unlocking}

Before focusing on the specific $M=2$ and $M=3$ cases, we start by discussing some general properties of the quantum walk of bounded particles, to clarify the differences with the single-particle counterpart. We consider the uniform-coupling regime; namely, $J_{j}=J, U_{j}=U, \mu_{j}=\mu$, in the initial state $|\{M\}, 1\rangle \propto\left(a_{1}^{\dagger}\right)^{M}|0\rangle$. The resulting effective interaction is

$$
H_{M}^{\text {eff }}=\left(\frac{J}{U}\right)^{M-1}\left(\begin{array}{ccccc}
B_{1}^{\text {eff }} & J_{1}^{\text {eff }} & & & \\
J^{\text {eff }} & B_{2}^{\text {eff }} & J^{\text {eff }} & & \\
& \ddots & \ddots & \ddots & \\
& & J^{\text {eff }} & B_{L^{-} 1}^{\text {eff }} & J^{\text {eff }} \\
& & & J^{\text {eff }} & B_{L}^{\text {eff }}
\end{array}\right) \text {, }
$$

where $J^{\text {eff }}=O(J), B_{1}^{\text {eff }}=B_{L}^{\text {eff }}=O\left[J(U / J)^{M-2}\right]$ while $B_{j}^{\text {eff }}$ for $j \neq 1, L$ is much smaller than $B_{1}^{\text {eff }}$ [boundary elements may be of order $O(J)$ or less while in the bulk they are even smaller]. The appearance of a larger effective field in the boundaries gives rise to a phenomenon which is called edge locking. Edge-locked states, which have been already described in Refs. [39,40] for $M \geqslant 3$, can be understood by using the theory of quasi-uniform tridiagonal matrices [60]. To describe this phenomenon we consider an initial wave packet localized in site 1 which evolves through the Hamiltonian (3) to the wave packet $|\psi(t)\rangle=\sum_{j}\left(e^{-i t H_{M}^{\text {eff }}}\right)_{j 1}|\{M\}, j\rangle$. Calling $H_{M}^{\text {eff }}=V E V^{\dagger}$ the spectral decomposition of the effective Hamiltonian, then $|\psi(t)\rangle=\sum_{k j} e^{-i t E_{k}} V_{1 k} V_{j k}^{*}|\{M\}, j\rangle$. Because of the mirror symmetry and for the properties of quasi-uniform matrices [60] one finds that $V_{L k}=V_{1 k}(-1)^{k} \approx$ $V_{1 k} e^{i L k}$ and $E_{k} \propto \cos (k)$ where $k$ is the quasimomentum, $k=k_{j}+O\left(L^{-1}\right)$, where $k_{j}=\pi j /(L+1)$ and $j=1, \ldots, L$. Therefore, the quantum walk of the bounded particle displays the standard expression of a wave-packet evolution [61], because $\langle\{M\}, L \mid \psi(t)\rangle=\sum_{k} e^{-i\left(t E_{k}-L k\right)}\left|V_{1 k}\right|^{2}$ where $\left|V_{1 k}\right|^{2}$ is the probability to excite the quasimomentum state $k$ by initializing the system in the first site. To simplify the theoretical analysis we assume that $B_{j}^{\text {eff }} \equiv B_{\text {bulk }}^{\text {eff }}$ is constant for $j \neq 1, L$ so, without loss of generality, we can set $B_{\text {bulk }}^{\text {eff }}=0$. Indeed, the Hamiltonian (3) and $H_{M}^{\text {eff }}-B_{\text {bulk }}^{\text {eff }} \mathbf{1}$ give rise to the same evolution aside from an irrelevant global phase. Within this description it is now clear that edge-locking appears when $B_{1}^{\text {eff }} \gg E_{k}$, since no quasimomentum state can be excited by initializing the system in a state where the bounded particle is in the first site (namely $\left|V_{1 k}\right|^{2} \approx 0$ for all the quasimomentum states). Indeed, in this regime this initialization excites out-of-band modes which are localized near the edges and do not propagate. As it is clear from Eq. (3), since $B_{1}^{\text {eff }}=B_{L}^{\text {eff }}=O\left[J(U / J)^{M-2}\right]$, the edge-locking condition $B_{1}^{\text {eff }} \gg E_{k}$ happens when $M \geqslant 3$, as obtained also in Ref. [39]. However, there is another form of quasilocking for $M=2$ which is not described in Ref. [39]. Indeed, for $M=2$ we find that $B_{1}^{\text {eff }}$ is of the same order of the energy band $E_{k}$ of the quasimomentum states and, as a consequence, the quasimomentum states with energy $B_{1}^{\text {eff }} \approx E_{k}$ are the ones involved by the dynamics. Since $E_{k} \propto \cos (k)$ when $B_{1}^{\text {eff }} \approx 0$ the relevant excitations consist mostly of quasimomentum 
states with almost-linear dispersion relation $\left(E_{k} \approx k\right.$ around $k=\pi / 2$ where $E_{\pi / 2} \simeq 0$ ). These states propagate without dispersion in the chain and therefore give rise to a high transmission quality. On the other hand, if $B_{1}^{\text {eff }} \neq 0$ other states with nonlinear dispersion relation are involved, which drastically lower the transmission quality. Because of this, we find that the state $|\psi\rangle=\left(a^{\dagger}\right)^{2}|0\rangle$ has a long delocalization time from the initial site during the relevant time $t^{*} \sim L U / J^{2}$.

Because edge localization is detrimental in quantum transfer applications, we analyze the possibility to "unlock" the states by compensating the energy gap between edge and bulk sites introducing a local static potential both in the first and the last site of the chain. Edge unlocking can be always obtained for any value of $M$ by adding suitable local chemical potentials $\mu_{j}$ around the edges such that the effective fields $B_{n}^{\text {eff }}$ are constant over the different sites $n$.

\section{B. Two particles}

In this section we analyze the dynamical behavior of a two-particle bound state. We first start from the uniform case, describe the edge unlocking, and then we consider how to engineer the couplings to maximize the transfer of a bound state and the generation of a NOON state.

\section{Edge unlocking}

For a uniform chain $J_{j}=J, U_{j}=U, \mu_{j}=\mu$ we find that the effective Hamiltonian $H_{\text {eff }}$ is the tridiagonal matrix in Eq. (2), where

$$
\begin{gathered}
J_{j}^{\text {eff }}=\frac{J^{2}}{2 U}, \\
B_{j}^{\text {eff }}=\left\{\begin{array}{lll}
\frac{J^{2}}{2 U}+U & \text { for } & j=1, L \\
\frac{J^{2}}{U}+U & \text { for } & j \neq 1, L .
\end{array}\right.
\end{gathered}
$$

The effect of the inhomogeneities in $B_{j}^{\text {eff }}$ is shown in Fig. 2 (top), where we analyze the dynamics of a bound particle initially in $|\psi(0)\rangle \propto\left(a_{1}^{\dagger}\right)^{2}|0\rangle$ in a uniform chain with $L=5$ and $U / J=5$. We study the probability $P_{i j}(t)$ that after the time $t$ one particle is in the site $i$ and the other is in the site $j$; namely, $P_{i j}=\frac{1}{1+\delta_{i j}}\left|\left\langle 0\left|a_{i} a_{j}\right| \psi(t)\right\rangle\right|^{2}$, where $|\psi(t)\rangle$ is the state evolved for a time $t$. In particular, we plot as a function of the time $t$ the probability to have the bound particle in the first site $P_{11}(t)$ and in the last site of the chain $P_{L L}(t)$. We observe that, despite the bound particle reaching the last site of the chain at the transfer time $t^{*} \sim L / J_{\text {eff }}$, the probability to be in the first site $P_{11}\left(t^{*}\right)$ is still not zero; namely, the delocalization time from the first site is slow compared with the transfer time $t^{*}$. As described in the previous section, this is due to the difference in effective energies $B_{j}^{\text {eff }}$ between the bulk and the edges which favors nonlinear excitations which, in turn, leads to a dispersive dynamics. This difference between the bulk and the edges can be made zero by adding two local chemical potentials at the endpoints, $\mu_{j}=-\beta^{\prime}\left(\delta_{j, 1}+\delta_{j, L}\right)$, where $\beta^{\prime}=J^{2} / 4 U$. As it can be seen in Fig. 2 (bottom), when the $\beta^{\prime}$ field is added, the delocalization time from the first time $P_{11}\left(t^{*}\right)$ [and consequently the transfer fidelity $P_{L L}\left(t^{*}\right)$ ] is strongly increased. We compare the results obtained for the transfer of a bound state with the propagation of a single particle in
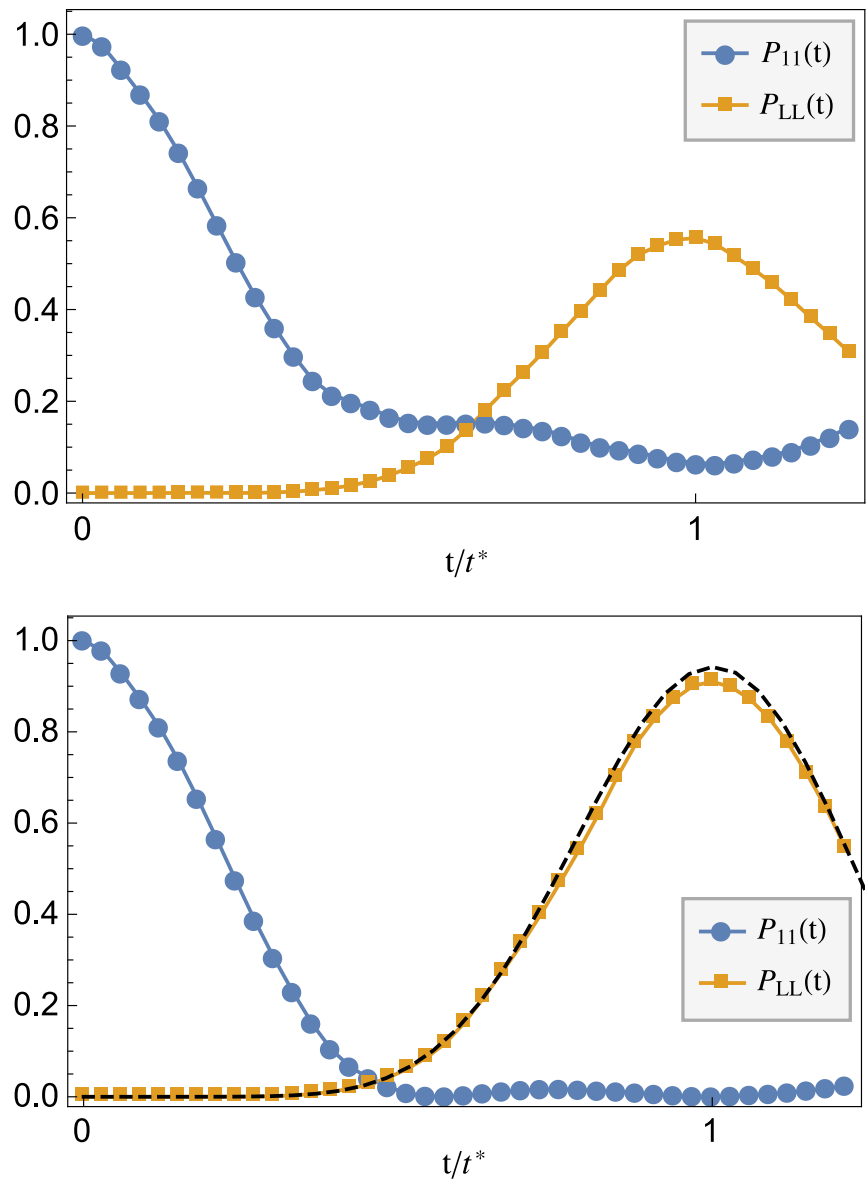

FIG. 2. Edge delocalization for a two-particle bound state: plot of the probability to have a bound particle in the first $P_{11}(t)$ and in the last $P_{L L}(t)$ site as a function of the time $t$, scaled for the transfer time $t^{*} \sim L / J_{\text {eff }}$ for a uniform chain with $L=5$ and $U / J=5$ in the initial state $|\psi(0)\rangle \propto\left(a_{1}^{\dagger}\right)^{2}|0\rangle$. We add a local field $\mu_{j}=-\beta^{\prime}\left(\delta_{j, 1}+\right.$ $\delta_{j, L}$ ) with strength $\beta^{\prime}=0$ (top), and $\beta^{\prime}=\beta_{\mathrm{opt}}^{\prime}=J^{2} / 4 U$ (bottom). To compare the results with the single-particle case we plot, with a dashed black line, the probability $P_{L}(t)=\mid\left\langle\left. 0\left|a_{L}\right| \psi(t)\right|^{2}\right.$ for a single particle initially in $a_{1}^{\dagger}|0\rangle$ (here $t^{*} \sim L / J$ ).

the lattice, initially in $a_{1}^{\dagger}|0\rangle$, by plotting in Fig. 2 (bottom) the probability $P_{L}(t)=\mid\left\langle\left. 0\left|a_{L}\right| \psi(t)\right|^{2}\right.$ (single-particle data are scaled for their transfer time $\left.t^{*} \sim L / J\right)$. The difference between the single-particle and the bound-particle results depends on the finite value of the interaction chosen $(U / J=5$ in Fig. 2). Indeed the agreements with single-particle behavior is very high as long as $U / J$ is large enough.

Because the effective model in Eq. (2) is valid in the regime $U / J \gg 1$, we analyze deviations from the theoretical value of $\beta^{\prime}$ by evaluating the dynamics with exact diagonalization techniques as in Refs. [37,38]. Once initialized the system in $|\psi(0)\rangle \propto\left(a_{1}^{\dagger}\right)^{2}|0\rangle$ we numerically find the value of $\beta^{\prime}$ that maximizes the probability to find, at the transfer time $t^{*} \sim$ $L / J_{\text {eff }}$, the bound particle in the last site of the chain $P_{L L}\left(t^{*}\right)$. We numerically find that, for a two-particle bound state, the optimal values of $\beta^{\prime}$ completely agree with the theoretical model $\beta^{\prime}=J^{2} / 4 U$ independently of the length of the chain, as long as $U / J \gtrsim 5$. 
When the hopping term $J$ and the onsite interaction $U$ have comparable amplitude, both the bound states and the single-particle states contribute to the dynamics [2]. We expect that, by increasing the onsite interaction, the effects of the free-particle states are reduced while the state transfer fidelity of a bound particle should converge to a constant value.

By analyzing $P_{L L}\left(t^{*}\right)$ we find that, when the optimal value for the localized field $\beta^{\prime}=J^{2} / 4 U$ is added in a uniform chain, values of the onsite interaction above $U / J \gtrsim 4$ guarantee an almost constant value of transfer fidelity for a two-particle bound state.

\section{Optimal state transfer of a two-particle bound state}

The state transfer efficiency of a two-particle bound state in a uniform chain can be improved by suitably tuning the tunneling couplings in the model in Eq. (1). Because a full engineering could be too demanding, here we consider the effect of a minimal engineering scheme [61,62], which consists of tuning the first and the last tunneling terms to $J_{1}=J_{L-1}=J_{0}$ while the rest of the chain has uniform couplings $J_{j}=J$. In this case the effective Hamiltonian (2) has effective interactions

$$
\begin{gathered}
J_{j}^{\text {eff }}= \begin{cases}\frac{J_{0}^{2}}{2 U} & \text { for } \quad j=1, L-1 \\
\frac{J^{2}}{2 U} & \text { for } \quad j \neq 1, L-1,\end{cases} \\
B_{j}^{\text {eff }}= \begin{cases}\frac{J_{0}^{2}}{2 U}+U & \text { for } \quad j=1, L \\
\frac{J_{0}^{2}}{2 U}+\frac{J^{2}}{2 U}+U & \text { for } \quad j=2, L-1 \\
\frac{J^{2}}{U}+U & \text { for } \quad j=3, \ldots, L-2 .\end{cases}
\end{gathered}
$$

To maximize the transfer fidelity one has then to remove the difference between the effective energies $B_{j}^{\text {eff }}$ and to optimize the values of $J_{j}^{\text {eff }}$ to achieve an optimal ballistic dynamics. Since the dynamics occurs in the effective subspace one can use the analytical theory presented in Ref. [61] to find the optimal value of $J_{0}^{\text {eff }} \equiv \frac{J_{0}^{2}}{2 U}$, given that the rest of the sites are coupled with a hopping strength $J^{\text {eff }} \equiv \frac{J^{2}}{2 U}$. Given the simple relationship between $J_{0}^{\text {eff }}$ and the strength $J_{0}$ of the Bose-Hubbard tunneling between the edges and the bulk, it is then straightforward to obtain $J_{0}$. Once $J_{0}$ is found, one needs to add local chemical potentials in both the first and the last two sites to remove the local energy difference in $B_{j}^{\text {eff }}$. By using our effective Hamiltonian expansion, we find that the state transfer is maximized by introducing two pairs of local fields $\mu_{j}=-\beta_{1}\left(\delta_{j, 1}+\delta_{j, L}\right)$ and $\mu_{j}=-\beta_{2}\left(\delta_{j, 2}+\delta_{j, L-1}\right)$, respectively, with strengths $\beta_{1}=\left(J_{0}^{2}-2 J^{2}\right) / 2 U$ and $\beta_{2}=$ $\left(J_{0}^{2}-J^{2}\right) / 2 U$. In Fig. 3 (top) we show the results obtained for the transfer fidelity $P_{L L}\left(t^{*}\right)$ as a function of $U / J$ when we use minimal engineering and the compensating fields $\beta_{1}$ and $\beta_{2}$. We observe a significant improvement of transfer fidelity compared with the results for a uniform chain. In Fig. 3 (bottom) we also highlight the difference between the single-particle dynamics and the bound-particle case for finite interaction $U$. As expected, for strong interparticle interaction $U$, a bound state behaves as a single-particle state. To highlight that minimal engineered schemes already have a significant impact in reducing the dispersion in the system, in Fig. 3 (bottom) we show the dynamics of a bound state in a lattice.
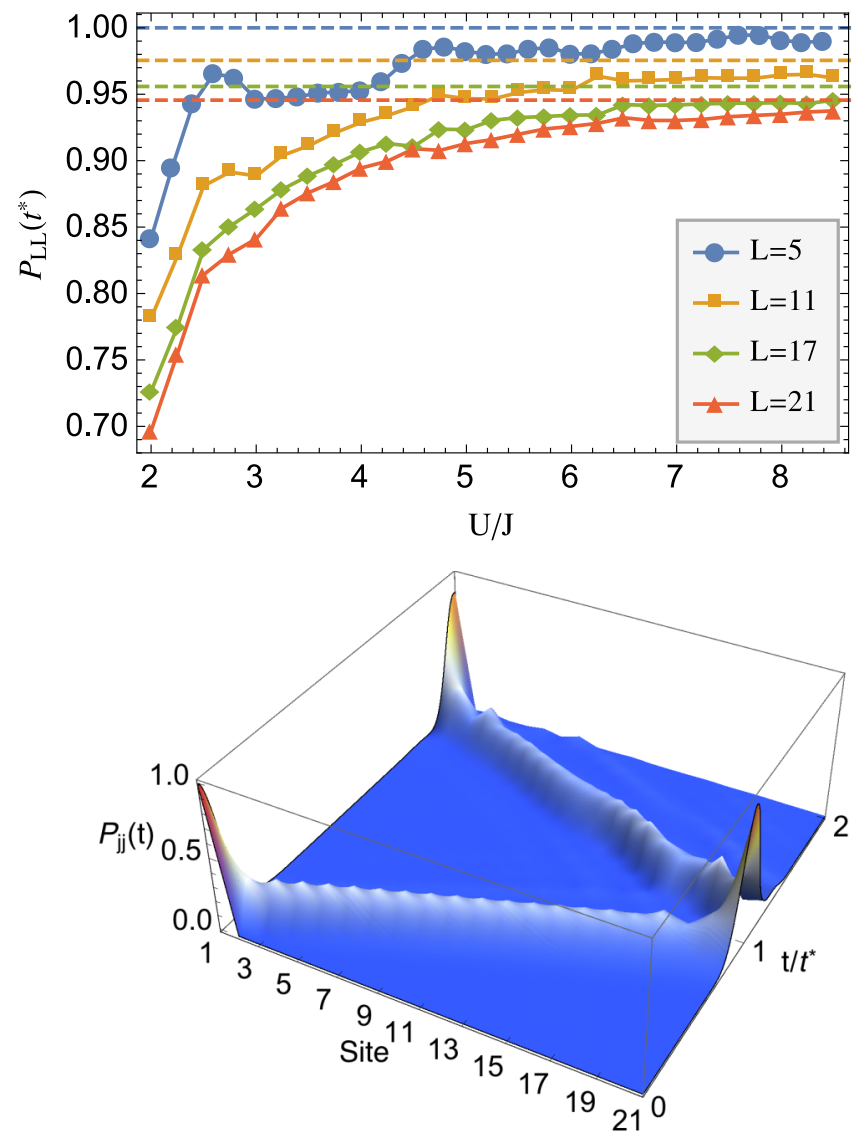

FIG. 3. Optimal transfer of a two-particle bound state: (top) Analysis of the transfer fidelity $P_{L L}\left(t^{*}\right)$ for the initial state $|\psi(0)\rangle \propto$ $\left(a_{1}^{\dagger}\right)^{2}|0\rangle$ as a function of the onsite interaction $U / J$ when the optimal transfer scheme $J_{1}=J_{L}=J_{0}$ and $J_{j}=J$ for $j \neq(1, L)$ is included in the model 1 . Here we also add two local impurities $\mu_{j}=-\beta_{1}\left(\delta_{j, 1}+\delta_{j, L}\right)$ and $\mu_{j}=-\beta_{2}\left(\delta_{j, 2}+\delta_{j, L-1}\right)$ where $\beta_{1}=\left(J_{0}^{2}-2 J^{2}\right) / 2 U$ and $\beta_{2}=\left(J_{0}^{2}-J^{2}\right) / 2 U$ to eliminate the edgelocking effect. The $J_{0}$ value is chosen by numerically maximizing the transfer fidelity in a single-particle manifold [61]. To compare the difference between a single particle and a bound state, we plot (with a dashed line) also the single-particle transfer fidelity $P_{L}\left(t^{*}\right)=\left|\left\langle 0\left|a_{L}\right| \psi\left(t^{*}\right)\right\rangle\right|^{2}$ obtained for a system initially in $a_{1}^{\dagger}|0\rangle$. (bottom) Probability $P_{j j}(t)$ to have a two-particle bound state in site $j$ at time $t / t^{*}$ for a minimal engineered chain with $L=21$ and $U / J=8$.

Specifically, we plot the probability $P_{j j}(t)=\left|\left\langle 0\left|a_{j}^{2}\right| \psi(t)\right\rangle\right|^{2} / 2$ to have a two-particle bound state in site $j$ as a function of the time $t / t^{*}$, for a minimal engineered chain with $L=21$ and $U / J=8$.

In analogous fashion the Bose-Hubbard Hamiltonian couplings can be tuned so that the effective Hamiltonian coincides with that allowing perfect state transfer [55], although this is much more demanding because it requires the engineering of all tunneling rates $J_{j}$ and all the chemical potentials $\mu_{j}$.

\section{Environmental effects}

State transfer schemes are generally robust against static imperfections in the couplings $[63,64]$. On the other hand, we explicitly test the robustness of our scheme against 

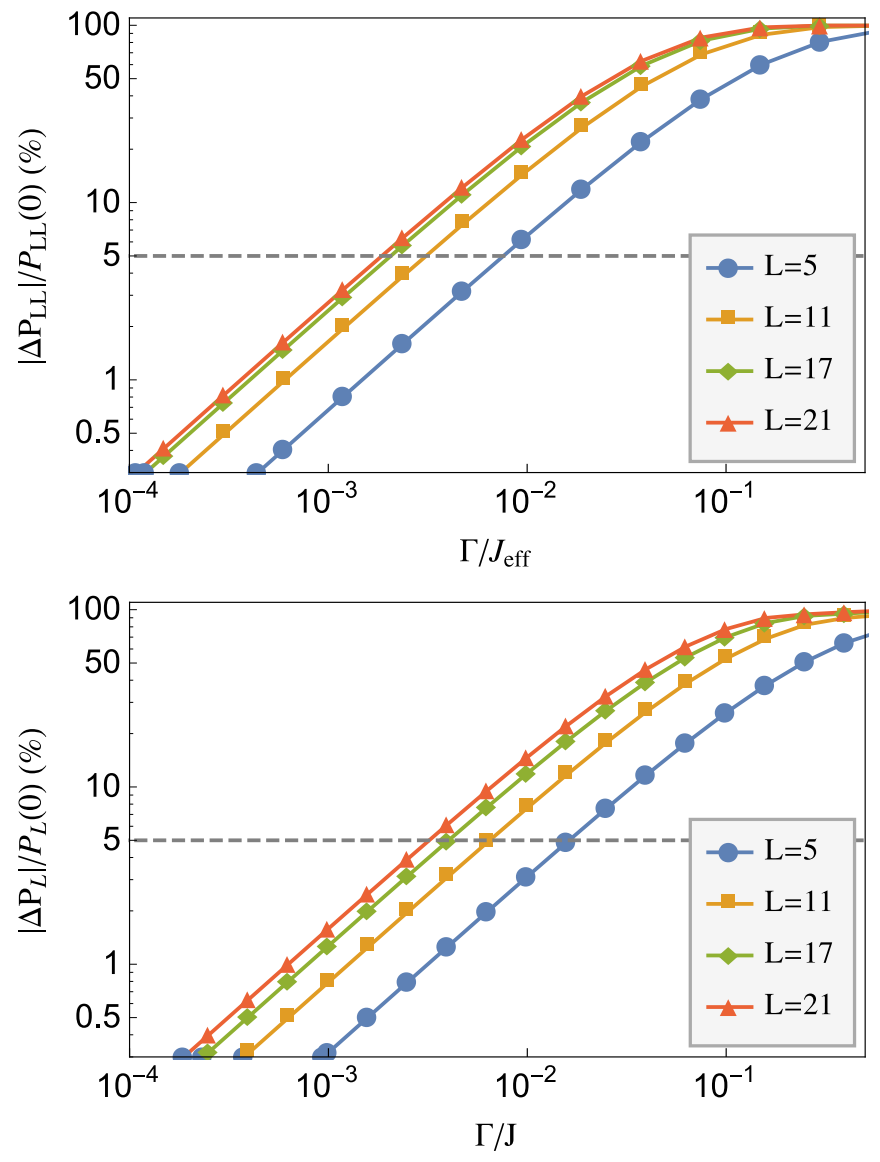

FIG. 4. Decoherence effects for a two-particle bound state: (top) Relative variation $\Delta P_{L L} / P_{L L}\left(t^{*}, \Gamma=0\right)$ of the transfer fidelity $P_{L L}\left(t^{*}\right)$ with respect to the case in absence of decoherence, for a uniform chain with $U / J=3$ and length $L$. Here $\Delta P_{L L}=$ $\left|P_{L L}\left(t^{*}, \Gamma\right)-P_{L L}\left(t^{*}, \Gamma=0\right)\right|$ and the dashed gray line is a threshold of a relative variation of the $5 \%$. Several chain lengths $L$ are considered. (bottom) Relative variation $\Delta P_{L} / P_{L}\left(t^{*}, \Gamma=0\right)$ for a single-particle state initially in $a_{1}^{\dagger}|0\rangle$.

dynamical environmental effect; specifically, dephasing due to spontaneous emission, which represents the main source of decoherence in optical lattices. The dynamics of the system in the lowest band is typically modeled as a master equation in Lindblad form [65-67]:

$$
\dot{\rho}=-i\left[H_{B H}, \rho\right]+\Gamma \sum_{i}\left(n_{i} \rho n_{i}-\frac{1}{2} n_{i} n_{i} \rho-\frac{1}{2} \rho n_{i} n_{i}\right) .
$$

Here $\Gamma$ is the effective scattering rate, and $H_{\mathrm{BS}}$ is the BoseHubbard Hamiltonian (1). We numerically solve Eq. (8) as shown in detail in Appendix C.

No relevant edge field optimal strength $\beta^{\prime}$ deviations are found when decoherence effects are introduced, for $\Gamma / J_{\text {eff }}<0.1$, where $J_{\text {eff }}=J^{2} / 2 U$. In Fig. 4 (top) we show how the transfer fidelity $P_{L L}\left(t^{*}\right)$ is affected as a function of the damping rate $\Gamma / J_{\text {eff }}$ in Eq. (8) for $U / J=3$. To better evaluate the difference with the zero-decoherence case, we show in Fig. 4 the relative variation $\left|\Delta P_{L L}\left(t^{*}\right)\right| / P_{L L}(0)=\left|P_{L L}(\Gamma)-P_{L L}(\Gamma=0)\right| / P_{L L}(\Gamma=0)$

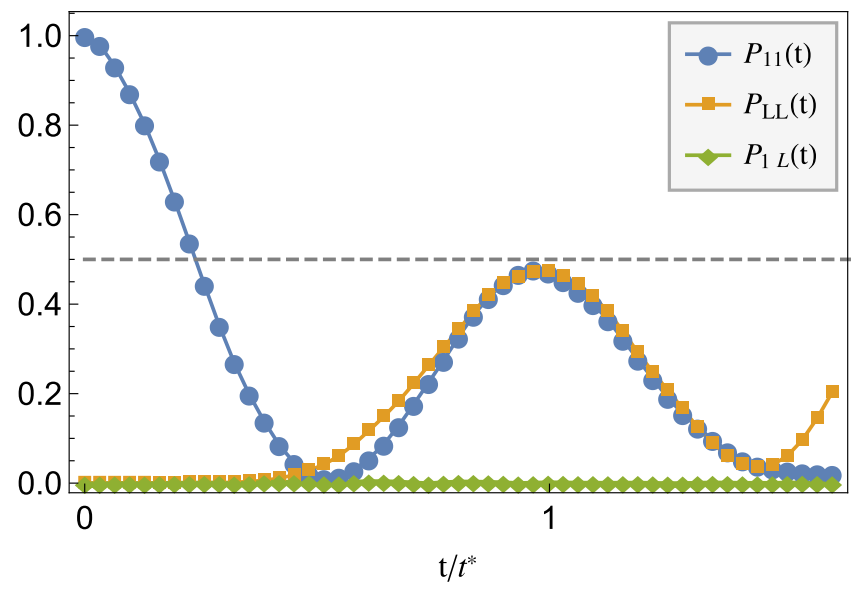

FIG. 5. Two-particle NOON state: plot of the probabilities $P_{i j}(t)$ to have one particle in site $i$ and the other in $j$, as a function of time, in units of the transfer time $t^{*}$, for two particles initially in $|\psi(0)\rangle \propto\left(a_{1}^{\dagger}\right)|0\rangle$. Here we consider a uniform chain with an impurity $\mu_{j}=-\beta \delta_{j, L / 2+1}$ where $\beta=0.789\left(J^{2} / 2 U\right)$ in a chain with $U / J=5$ and length $L=5$. The absence of the $P_{1 L}\left(t^{*}\right)$ term is evidence that the output state at $t=t^{*} \simeq U L / J^{2}$ is the NOON state with two particles. The gray dashed line represents the results for an ideal lossless NOON state generation.

with respect to the no-decoherence case, as a function of the damping parameter $\Gamma / J_{\text {eff }}$. We observe deviations of less than the $5 \%$ for $\Gamma / J_{\text {eff }} \simeq 10^{-2}-10^{-3}$ for chain lengths between $L \in\{5, \ldots, 21\}$ which are typical values for blue detuned optical lattices [66,67]. In Fig. 4 (bottom) we show the effects of the decoherence in the state transfer fidelity for a single-particle state, initially in $a_{1}^{\dagger}|0\rangle$.

\section{NOON state generation with a two-particle bound state}

In this section we consider an imperfect fractional revival by considering a uniform evolution, although the present results can be extended with a further engineering to achieve perfect fractional revivals.

We consider the simplest scheme where the wave-packet splitting is achieved by using a local barrier in the middle of the chain, as shown in Fig. 1 and discussed in Ref. [37]. We set the value $\beta^{\prime}=J^{2} / 4 U$ for the edge fields $\mu_{j}=-\beta^{\prime}\left(\delta_{j, 1}+\delta_{j, L}\right)$ to remove edge locking. Then we add a local field in the middle of the chain $\mu_{j}=-\beta \delta_{j, L / 2+1}$ to trigger a wave-packet splitting [37]. Indeed, the extra barrier favors the splitting of the propagating bound particle wave packet into a transmitted and reflected component. It has been shown in Ref. [37] that, for a single-particle quantum walk, the optimal 50-50 splitting is obtained when the strength $\beta$ of this extra barrier is equal to the hopping rate. We expect that, when the bound particle impinges the splitting field because it behaves like an effective single particle, the output state, measured at the endpoints, will be $\left|\psi\left(t^{*}\right)\right\rangle_{1 L}=\frac{1}{\sqrt{2}}(|2,0\rangle+i|0,2\rangle)$; namely, we generate a NOON state with two particles [here $|2\rangle=\left(a^{\dagger}\right)^{2}|0\rangle / \sqrt{2}$ ]. On the other hand, if the two particles are noninteracting $U / J=0$, the effect of the splitting field is to produce also a nonzero probability $P_{1 L}\left(t^{*}\right)$ to have one particle in each end [68]. We show for a $L=5$ chain with $U / J=5$ in Fig. 5 that, 


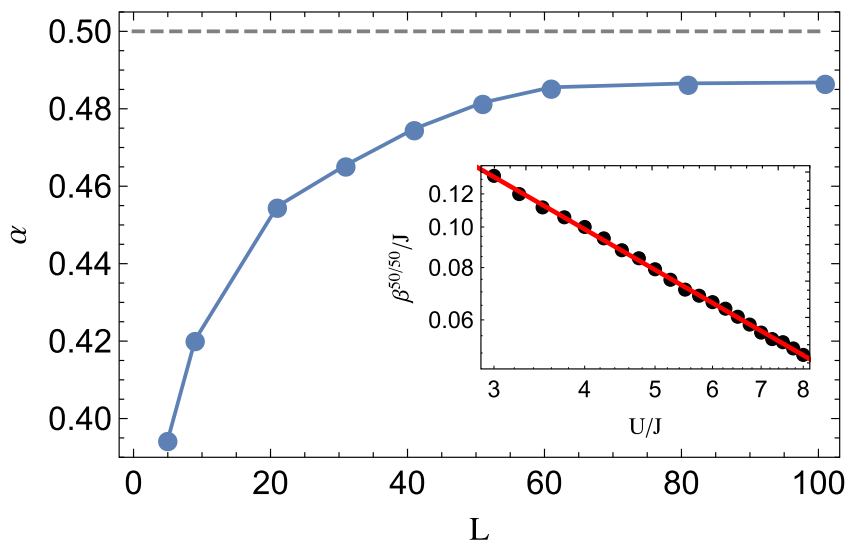

FIG. 6. Finite-size effects in the two-particle NOON state generation: analysis of the scaling factor $\alpha$ where $\beta^{50-50}=\alpha J^{2} / U$, as a function of the chain length $L$ for generating a two-particle NOON state. The gray line represents the theoretical results from the effective Hamiltonian theory, which holds for $L \gg 1$. (inset) Analysis of the optimal value of $\beta$ of the local field $\mu_{j}=-\beta \delta_{j, L / 2+1}$ which produces the NOON state with two particles as a function of $U / J$ for $L=5$. The red line is the fit $\beta=\beta^{50-50}=0.395 \mathrm{~J}^{2} / U$.

for a bound particle, that term is suppressed at the transfer time $t^{*}$, as expected from a bound-particle effective evolution. Therefore, we can conclude that the output state is, when $U$ is large enough, the NOON state with two particles, apart from a damping factor due to dispersion. By performing an effective Hamiltonian expansion in the onsite interaction term we show that to have a balanced splitting in the effective space, the strength of the splitting field in the real chain must be $\beta=\beta^{50-50}=J^{2} / 2 U$, in the limit $L \gg 1$.

Finite-length corrections are found numerically by finding the $\beta$ value for which the difference $P_{11}\left(t^{*}\right)-P_{L L}\left(t^{*}\right)$ is zero. As shown in the inset in Fig. 6, for a $L=5$ uniform chain, $\beta^{50-50}$ scales as $1 / U$. The finite length factors, found from a fit over the data for several chain lengths, are shown in Fig. 6. By increasing the chain length $L$ the $\beta^{50-50}$ values are closer to $0.5 J^{2} / U$, in agreement with the effective Hamiltonian analysis. We underline that the NOON state creation efficiency can be made arbitrarily close to $100 \%$ by tuning the couplings in the effective bound particle subspace by using the techniques for perfect splitting developed in Refs. $[38,58]$ which require a complete engineering of the couplings in the Hamiltonian (1). In Sec. IV we propose two methods for detecting the NOON state generated by measuring interference fringes.

\section{Even chains}

Here we clarify that our scheme is not limited to odd length chains but also can be applied to even chains by tuning both the middle tunneling coupling strength $J_{L / 2}$ and adding two pairs of local fields $\mu_{j}=-\beta_{1}\left(\delta_{j, 1}+\delta_{j, L}\right)$ and $\mu_{j}=-\beta_{2}\left(\delta_{j, 2}+\delta_{j, L-1}\right)$, respectively, in the Hamiltonian (1). Using the results in Ref. [37] for the splitting of a single particle we find, from the effective Hamiltonian model, that the optimal coupling strengths to generate a two-particle NOON state between the endpoints of a uniform chain are $J_{L / 2}=J(\sqrt{2}-1)^{1 / 2}, \beta_{1}=J / 4 U$, and $\beta_{2}=J(2-\sqrt{2}) / 4 U$, respectively.

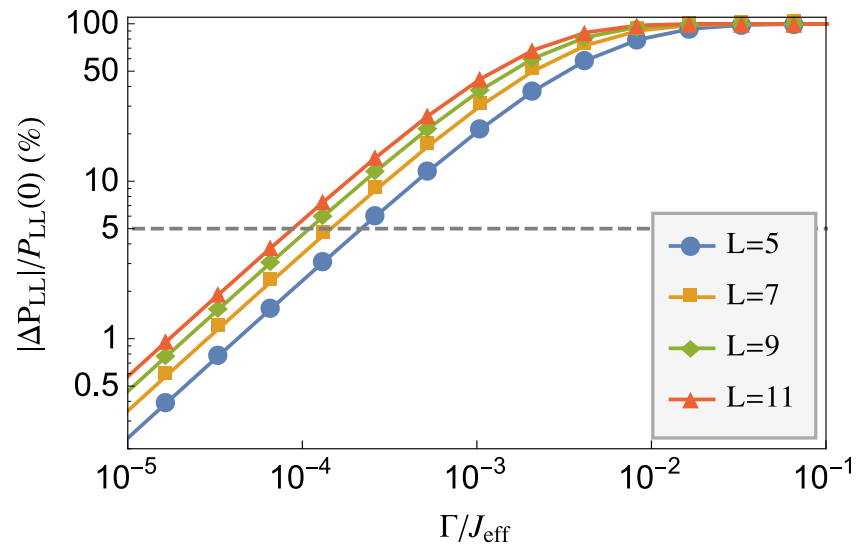

FIG. 7. Decoherence effects for a three-particle bound state: relative variation $\Delta P_{L L} / P_{L L}\left(t^{*}, \Gamma=0\right)$ with respect to decoherence-free case, for a uniform chain with $U / J=2$. Here $\Delta P_{L L}=\mid P_{L L}\left(t^{*}, \Gamma\right)-$ $P_{L L}\left(t^{*}, \Gamma=0\right) \mid$ and the dashed gray line is a threshold of a relative variation of the $5 \%$. Several chain lengths $L$ are considered.

\section{Three particles}

The extension of the previous scheme to more-than-twoparticle bound states enhances its nonclassical state generation capabilities; namely, towards realizing small cat states between remote sites. As before, the onsite interaction generates bound states with three particles when initially located in the same site. Similarly to the two-particle case, one would expect that the results of the splitting process, when the onsite interaction is strong enough, is to produce a NOON state with $N=3$.

As for the two-particle case, for large onsite interactions the effective evolution in the bound-particle subspace is described by Eq. (2). We consider a uniform chain, while a minimally engineered model is discussed in Appendix B. For a uniform chain the effective hopping is $J_{\text {eff }}=3 J^{3} / 16 U^{2}$. Moreover, to remove edge locking and compensate the energy gap between the endpoint sites and the bulk of the chain we have to introduce two local fields $\mu_{j}=-\beta^{\prime}\left(\delta_{j, 1}+\delta_{j, L}\right)$. From our expansion we find for a uniform chain that $\beta^{\prime}=J^{2} / 8 U$. To check for finite-size correction to the above analytical prediction we numerically analyze the value of $\beta^{\prime}$ for several chain lengths $L$ for the initial state $|\psi(0)\rangle \propto\left(a_{1}^{\dagger}\right)^{3}|0\rangle$ as a function of the onsite interaction $U$. We find that with high accuracy the estimated field $\beta^{\prime}=J^{2} / 8 U$ is independent of $L$. We analyze the probability $P_{L L L}\left(t^{*}\right)$ to have three particles in the site $L$ after time $t^{*}$ for a uniform chain as a function of the onsite interaction. We find that values of the onsite interaction above $U / J \gtrsim 4$ guarantee an almost constant value of transfer fidelity for chain lengths $L \in\{5, \ldots, 21\}$.

In Fig. 7 we show the effect of decoherence due to spontaneous emission, Eq. (8), for several chain lengths $L$ as a function of the decoherence rate $\Gamma / J_{\text {eff }}$ where $J_{\text {eff }}=$ $3 J^{3} / 16 U^{2}$. We observe relative variation of less than the $5 \%$ with respect to the decoherence-free case, for $\Gamma / J_{\text {eff }} \lesssim 1.3 \times$ $10^{-4}$ up to $L=7$ sites. The state transmission fidelity of a three-bound-particle state can be optimized by engineering the end tunneling couplings of the chain, as shown in Appendix B. In this case to bypass the edge-localization effects we also need 


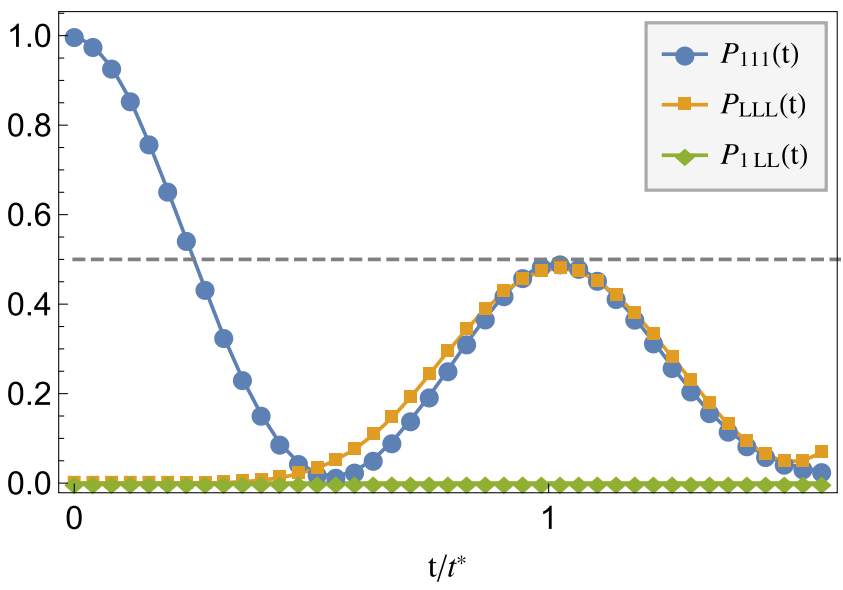

FIG. 8. Three-particle NOON state: Joint probabilities $P_{i j k}(t)$ as a function of the time, in units of the transfer time $t^{*}$, for three particles initially in $|\psi(0)\rangle \propto\left(a_{1}^{\dagger}\right)|0\rangle$ for a uniform chain with an impurity $\beta=0.099 J^{3} / U^{2}$ and $\beta^{\prime}=J^{2} / 8 U$ in the middle of the chain with $U / J=5$ and length $L=5$. The absence of the $P_{1 L L}(t)$ term is evidence that the output state at $t=t^{*}$ is the NOON state with two particles. The gray dashed line represents the results for an ideal lossless NOON state generation. We found also that $P_{1 L L}(t)=P_{11 L}(t)$.

to add a local chemical-potential tuning in the first two and last two sites of the chain.

\section{NOON state generation for a three-particle bound state}

In the noninteracting case $U / J=0$ when three particles are initially located in the first site $|\psi(0)\rangle \propto\left(a_{1}^{\dagger}\right)^{3}|0\rangle$ an ideal beam splitter transformation generates as output a state with probabilities [68] $P_{111}=P_{L L L}=1 / 8, \quad P_{1 L L}=P_{11 L}=3 / 8$ where we define

$$
P_{j k l}(t)=\frac{\left|\left\langle 0\left|a_{i} a_{j} a_{k}\right| \psi(t)\right\rangle\right|^{2}}{1+\delta_{i j}+\delta_{j k}+\delta_{i k}+2 \delta_{i j} \delta_{j k}}
$$

as the probability to have the three particles in the sites $i, j, k$. We expect that, when the onsite interaction is strong enough, the bound particle behaves as an effective single bound particle, thus the terms $P_{1 L L}, P_{11 L}$ are suppressed and the output state at the endpoints effectively results in the NOON state $\left|\psi\left(t^{*}\right)\right\rangle_{1 L}=\frac{1}{\sqrt{2}}(|3,0\rangle+i|0,3\rangle)$, where $|3\rangle=$ $\left(a^{\dagger}\right)^{3}|0\rangle / \sqrt{6}$. In Fig. 8 we plot, as a function of time (in $t^{*} \simeq L / J_{\text {eff }}$ units) the probability to have a three-particle bound state, respectively, in the first site $P_{111}(t)$, in the last $P_{L L L}(t)$, and one particle in the first site and two in the last $P_{1 L L}(t)$ in a uniform chain with $U / J=5$ and $L=5$. Here we set the edge-field strength to $\beta^{\prime}=J^{2} / 8 U$ and we find numerically that the splitting field to have a balanced splitting is $\beta=\beta^{50-50}=0.099 \mathrm{~J}^{3} / U^{2}$. The absence of the term $P_{1 L L}\left(t^{*}\right)=P_{11 L}\left(t^{*}\right)$ is evidence that a NOON state with three particles is generated between the edges of the chain. From the effective Hamiltonian description we find that to generate a balanced splitting of a bound three-particle wave packet, we need to add a local field $\mu_{j}=-\beta^{50-50} \delta_{j, L / 2+1}$ whose strength, when $L \gg 1$, is $\beta^{50-50}=J^{3} / 8 U^{2}$ (as explained in Appendix B). However, finite-size corrections change the

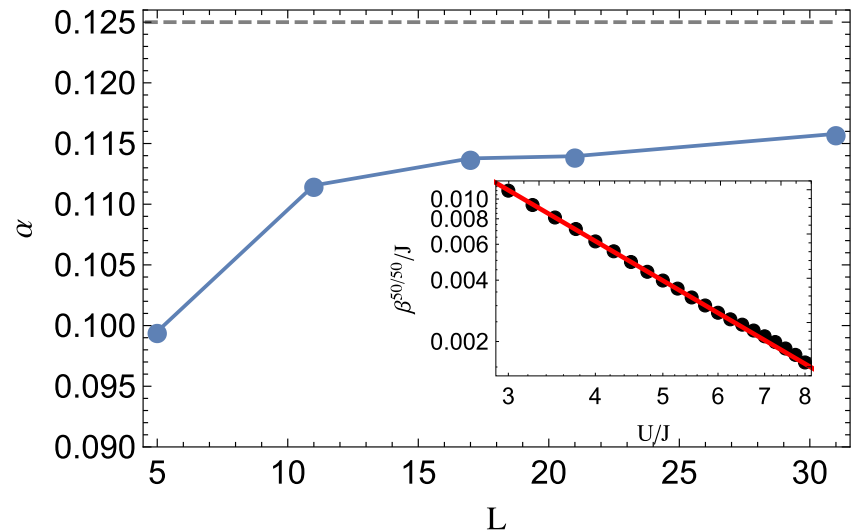

FIG. 9. Finite-size effects in the three-particle NOON state generation: Analysis of the scaling factor $\alpha$ as a function of chain length $L$ for a three-particle bound state, where $\beta^{50-50}=\alpha J^{3} / U^{2}$. The dashed gray line represents the theoretical value from the effective Hamiltonian description. (inset) Analysis of the optimal value of $\beta$ to produce the NOON state with three particles as a function of $U / J$ in a uniform chain with length $L=5$. The red line is the fit $\beta^{50-50}=\alpha J^{3} / U^{2}$ where $\alpha=0.099$.

value of $\beta^{50-50}$ and, by performing a numerical fit over the data for a uniform chain with $L=5$ (whose results are shown in the inset of Fig. 9), we find that $\beta^{50-50}$ scales with the onsite interaction as $\beta^{50-50}=\alpha J^{3} / U^{2}$ where $\alpha \simeq 0.099$. Deviations from the theoretical value of $\beta^{50-50}=J^{3} / 8 U^{2}$, which are shown in Fig. 9, have been found by analyzing the results obtained for several chain lengths $L$. Here the dashed gray line represents the theoretical value of the coefficient $\alpha$ of the splitting field for $L \gg 1$.

\section{NOON STATE VERIFICATION}

The creation and the detection of a NOON state can be revealed by measuring the interference fringes in a MachZehnder setup. After initializing the bound particles in the initial state $|\psi(0)\rangle \propto\left(a_{1}^{\dagger}\right)^{N}|0\rangle$, the NOON state is generated by the splitting field in the middle of the chain, as previously discussed. By freezing the dynamics of the system at the transfer time $t^{*}$, a controllable phase factor can be added by using a local field in the last site of the chain. Finally, once lowered the lattice potential, a second beam splitter operation is performed by the splitting field, which produces interference fringes at the endpoints of the chain at $2 t^{*}$.

For an ideal lossless transformation the state at the two boundary sites of the chain at the transfer time $t^{*}$ would be

$$
\left|\psi\left(t^{*}\right)\right\rangle_{1 L}=\frac{1}{\sqrt{2}}(|N 0\rangle+i|0 N\rangle) .
$$

Once we apply the phase transformation $\Phi=\operatorname{diag}\left(1, e^{i \phi}\right)$ (namely, a phase shift on site $L$ ), a second ideal beam-splitting transformation would produce the output state at time $2 t^{*}$ :

$$
\left|\psi\left(2 t^{*}\right)\right\rangle_{1 L}=\frac{1}{2}\left[\left(1-e^{i N \phi}\right)|N 0\rangle+i\left(1+e^{i N \phi}\right)|0 N\rangle\right],
$$

where the phase accumulated is $N \phi$ with $N$ being the number of particles in the NOON state. Therefore, the presence of a NOON state is revealed by measuring the interference 


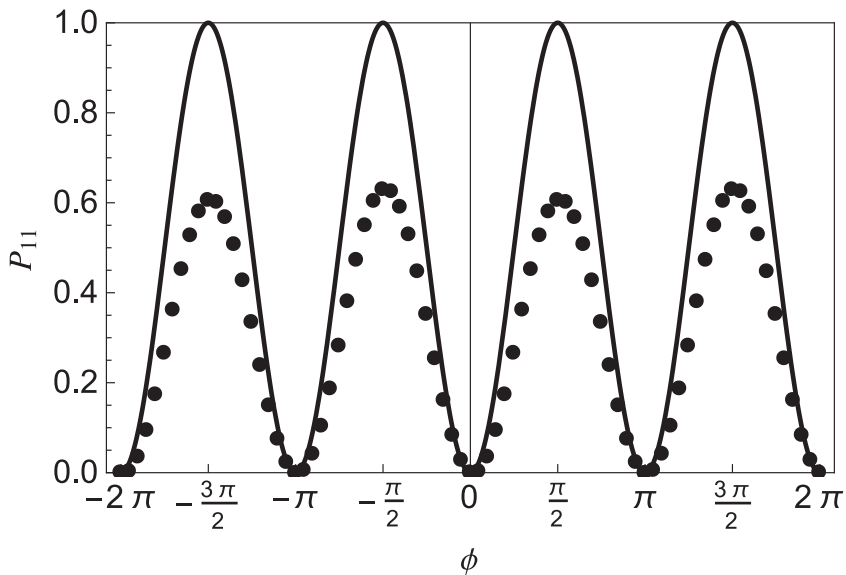

FIG. 10. NOON state detection with bound particles: Interference fringes in a Mach-Zehnder scheme for a two-particle bound state for a chain with $L=5$ and $U / J=5$. We plot the probability $P_{11}\left(t^{*}\right)$ to have the bound-state particle in the first site after a time evolution for $t^{*} \simeq L U / J^{2}$, when a phase factor $\phi$ is introduced in the system. The line data represent the result for an ideal lossless Mach-Zehnder transformation.

fringes (i.e., the probability to have $N$ particles in the first site as a function of $\phi$ ). Although previous experimental results measured just the parity of single sites (which would exclude a direct observation of the $N=2$ case discussed so far), this detection issue in optical lattice has been recently circumvented up to four particles in the same site [12].

We evaluate numerically the interference fringes for a twoparticle bound state in a uniform chain with length $L=5$ and $U / J=5$. To introduce a controllable phase factor between the endpoints of the chain we freeze the dynamics at time $t^{*} \simeq L U / J^{2}$ by increasing the lattice potential depth, then we apply a local field in the last site. The Hamiltonian (1) is then quenched at $t^{*}$ to

$$
H^{\prime}=\sum_{j=1}^{L} U n_{j}\left(n_{j}-1\right)-\beta_{L} n_{L} .
$$

We let the system evolve for a time $t^{\prime}$ and the phase difference generated between site $L$ and 1 is $\phi=\beta_{L} t^{\prime}$. Then, for $t>t^{\prime}$, the lattice potential is lowered again and the dynamics is described again by the Hamiltonian (1). Finally we let the system evolve and we evaluate the probability $P_{11}$ to have the bound particle in the first site at the transfer time. An alternative approach, discussed in Ref. [37] for the single-particle case, is to add a further step-like potential on the right half of the effective chain, which corresponds to a piecewise constant potential in the Bose-Hubbard model.

In Fig. 10 we show the results for $P_{11}$ as a function of the phase factor $\phi$. By comparing our data with the results of an ideal lossless transformation (line in Fig. 10) we observe that the interference fringes are in the same positions as in the ideal transformation. The influence of the chain dispersion reduces the height of the peaks compared with the ideal case. However, the efficiency of this scheme can be pushed up to $100 \%$ by engineering the chain couplings $[37,38]$ in the effective subspace of bound particles. This method can

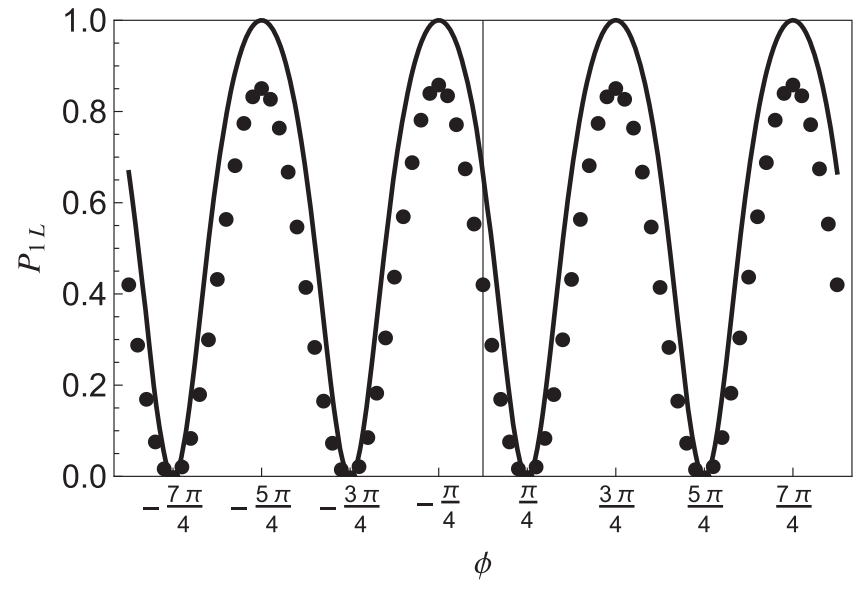

FIG. 11. NOON state detection after quenched interparticle interaction: interference fringes after the quench to $U / J=0$. The chain has length $L=5$ and we set $U / J=5$ for generating the two-particle NOON state in the edges at time $t^{*} \simeq L U / J^{2}$. Once the NOON state is generated the dynamics is frozen by increasing the lattice depth, then a controllable phase factor $\phi$ is added by tilting the lattice. Once the interparticle interaction is quenched to $U / J=0$ we let the system evolve and we measure the probability $P_{1 L}\left(t^{\prime \prime}\right)$ where $t^{\prime \prime} \simeq L / J$ is the transfer time of the free chain.

be easily extended to bound states with a higher number of particles.

An alternative approach to detect the NOON state for $N=2$ is to quench the interparticle interaction $(U / J=0$, i.e., via Feshbach resonances) just after the phase factor is added in the system. In the lossless case the final state of the two boundary sites is

$$
\begin{aligned}
\left|\psi\left(t^{\prime \prime}\right)\right\rangle_{1 L} \propto & {\left[\left(1-i e^{i N \phi}\right)|20\rangle+\left(i e^{i N \phi}-1\right)|02\rangle\right.} \\
+ & \left.2 i\left(1+e^{i N \phi}\right)|11\rangle\right] .
\end{aligned}
$$

Here $t^{\prime \prime}$ is the transfer time of free particles in the lattice $t^{\prime \prime} \simeq L / J$. The probability to find one particle in each end at $t^{\prime \prime}$ is

$$
P_{1 L}\left(t^{\prime \prime}\right)=\frac{2(\sin N \phi-1)}{\sin N \phi-3} .
$$

From the latter we see with a choice of $\phi=-5 \pi / 4$ the output state results in $\left|\psi\left(t^{\prime \prime}\right)\right\rangle_{1 L}=|11\rangle$, which can be measured by using single-particle fluorescence techniques. The latter scheme has two main advantages: first of all it circumvents the parity projection measurement issue, because the fringes measurement requires only single-atom detection. In second place the decoherence influence is reduced because, after the phase factor is added, it exploits free particle propagation, which is faster compared with the bound-state case. In Fig. 11 we show the results obtained for the probability to observe one particle in each end, in a chain with $L=5$ and $U / J=5$, at time $t^{\prime \prime}$, compared with the lossless case in Eq. (12).

\section{QUANTUM ENHANCED METROLOGY}

As shown in Fig. 10 the interference fringes using a $M=2$ NOON state have half the spacing compared with the single-particle case. This gives rise to a larger slope of 
the probabilities as a function of $\phi$ that, in turn, enables the estimation of the phase $\phi$ from the measurements with higher sensitivity [18].

This argument can be made more precise by computing the quantum Fisher information $F_{Q}$, which provides a lower bound on the variance of an estimator $\hat{\phi}$ of the phase $\phi$ via the Cramér-Rao bound $(\Delta \hat{\phi})^{2} \geqslant 1 /\left(\nu F_{Q}\right)$, where $v$ is the number of independent measurements. By the law of large numbers $\Delta \hat{\phi}$ decreases as $1 / \sqrt{v}$ for increasing $v$. On the other hand, NOON states with $M$ particles provides a quantum enhanced sensitivity for phase estimation with a variance that decreases as $M^{-1}$. This scaling is obtained from the evaluation of the quantum Fisher information $F_{Q}$ that for pure states is $[69,70]$ :

$$
F_{Q}=4\left[\left\langle\psi^{\prime}(\phi) \mid \psi^{\prime}(\phi)\right\rangle-\left|\left\langle\psi^{\prime}(\phi) \mid \psi(\phi)\right\rangle\right|^{2}\right],
$$

where $\left|\psi^{\prime}(\phi)\right\rangle=\partial|\psi(\phi)\rangle / \partial \phi$. In our case the NOON state is generated by letting the initial state $\left|\psi_{t=0}\right\rangle \propto\left(a_{1}^{\dagger}\right)^{M}|0\rangle$ evolve for $t \simeq L U^{M-1} / J^{M}$. A relative phase factor between the endpoints is then added, as described in the previous section, by using a local field in the last site of the chain. After these steps we get then the state $|\psi(\phi)\rangle=\exp \left(-i n_{L} \phi\right)\left|\psi_{t}\right\rangle$ which, in the ideal case, would be a $\phi$-dependent NOON state $\left(|M 0\rangle_{1 L}+i e^{-i \phi M}|0 M\rangle_{1 L}\right) / \sqrt{2}$ on sites $1, L$. Therefore, in general, from Eq. (13) we get

$$
F_{Q}=4 \Delta n_{L}^{2}=4\left(\left\langle n_{L}^{2}\right\rangle-\left\langle n_{L}\right\rangle^{2}\right),
$$

which in the ideal case results in $F_{Q}=M^{2}$. In our scheme, the ideal quantum limit can be achieved by using a fully engineered chain [38] which enables the creation of the ideal NOON state. This demonstrates the quantum enhanced sensitivity provided by ideal NOON states.

We now show that even the imperfect NOON states obtained with uniform chains are sufficient to achieve a quantum enhanced sensitivity. We consider a uniform chain with length $L=5$ and a bound state with $M=2,3$. In Fig. 12 we plot the best achievable phase uncertainty $\Delta \phi=1 / \sqrt{F_{Q}}$, in a single measurement $v=1$, as a function of the onsite interaction $U / J$ for a two and a three bound state. The gray and the red lines

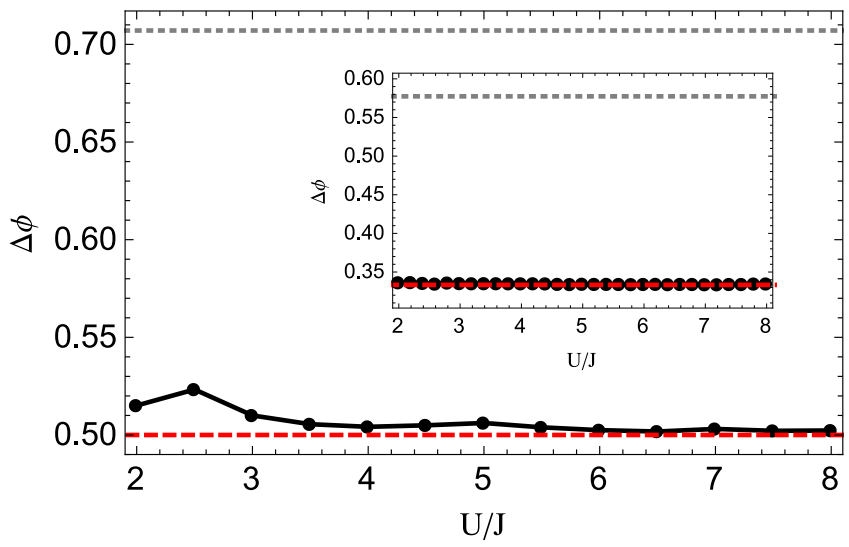

FIG. 12. Phase estimation precision $\Delta \phi=1 / \sqrt{F_{Q}}$, where $F_{Q}$ is the quantum Fisher information for the estimator $n_{L}$ in a uniform chain with $L=5$ respectively for a two-particle bound state and for a three-particle bound state (inset) as a function of the onsite interaction $U / J$. The red dashed (gray) dotted lines represent the ideal quantum (classical) lower bound $\Delta \phi_{\text {quant }}=1 / M\left(\Delta \phi_{\mathrm{cl}}=1 / \sqrt{M}\right)$. represent respectively the "classical" limit $\Delta \phi_{\mathrm{cl}}=1 / \sqrt{M}$ (obtained, e.g., by using coherent states where $M$ is the average number of particles) and the ideal quantum limit $\Delta \phi_{\text {quant }}=1 / M$. The black line on the other hand represents Eq. (14). Both for two- and three-particle bound states we observe an improvement in the phase estimation precision compared with the classical case, which is quite close to the ideal limit for an ideal NOON state and increases with the onsite interaction $U / J$.

\section{CONCLUSION}

In this paper we analyze the possibility to transfer states of bound particles between the endpoints of finite lattice and their use for small cat state (NOON state) generation, using a minimal control setup.

We derive an effective single-particle theory for the dynamics of a bound-particle state in a Bose-Hubbard model with tunable couplings by using an accurate effective Hamiltonian technique. By introducing suitable static local impurities in the edges of the lattice potential we show how to inhibit edgelocalization effects and enable the bound-state dynamics. This allows us to realize transformations between far sites, even when strongly interacting particles are involved. Specifically, we show how state transfer coupling schemes (in particular minimal engineering schemes), developed for single-particle states, can be introduced in our model to improve the efficiency. We then show how to split the propagating bound-state wave function to generate cat states (NOON states) between the endpoints of finite lattices with high fidelity, in a minimal control setup, by tuning a single local field in the middle of the chain. We analyze also how environmental effects affect our scheme; namely, taking into account decoherence due spontaneous emission in an optical lattice setup, finding the parameters' regime in which our scheme is robust.

Our model is of interest for state transfer application with $N \geqslant 2$ strongly interacting particles and for metrology applications. In particular, compared with other systems, it can provide some advantages for sensing external local fields in a Mach-Zehnder configuration. Indeed, we specifically show that, even in a uniform chain, the obtained NOON states give an improvement for the phase estimation between the output arms of an interferometer. Moreover, our method can be straightforwardly extended to fully engineered chains to realize $100 \%$ fidelity operations between distant sites, such as the perfect state transfer of bound-particle states or the perfect NOON state generation in an arbitrary long chain. As a future perspective, it will be interesting to adapt pumping techniques [71] or tunneling modulation techniques [72] to speed up the transfer time and thus enable the creation of higher NOON states compatible with the coherence time of the system.

\section{ACKNOWLEDGMENTS}

The research leading to these results has received funding from the European Research Council under the European Union's Seventh Framework Programme (FP/20072013)/ERC Grant Agreement No. 308253. The authors thank 
Marco Genoni, Gerald Milburn, Hendrik Weimer, and Luca Marmugi for interesting discussions and suggestions.

\section{APPENDIX A: EFFECTIVE HAMILTONIAN}

For the sake of clarity and self-consistency, here we describe the effective Hamiltonian theory which has been derived and successfully applied in Ref. [45].

We assume that the Hilbert space is divided into two subspaces, the effective subspace and the irrelevant subspace, which are well separated in energy when the interaction strength $U$ is much greater than the chemical potential and the tunneling rates. We define a projection operator $\mathbb{P}$ which projects the states to the relevant subspace and the complementary operator $\mathbb{Q}=\mathbf{1}-\mathbb{P}$. Because $\mathbb{P}$ and $\mathbb{Q}$ operate on disconnected subspaces we have

$$
\begin{aligned}
\mathbb{P}+\mathbb{Q} & =\mathbf{1}, \\
\mathbb{P} \mathbb{Q} & =\mathbb{Q} \mathbb{P}=0, \\
\mathbb{P}^{2} & =\mathbb{P}, \\
\mathbb{Q}^{2} & =\mathbb{Q} .
\end{aligned}
$$

Once Eqs. (A1) are applied on the Schrödinger equation, one obtains a system of two coupled equations for the dynamics in the relevant and irrelevant subspaces:

$$
\begin{aligned}
i \partial_{t} \mathbb{P}|\psi\rangle & =(\mathbb{P} H \mathbb{P}+\mathbb{P} H \mathbb{Q})|\psi\rangle, \\
i \partial_{t} \mathbb{Q}|\psi\rangle & =(\mathbb{Q} H \mathbb{P}+\mathbb{Q} H \mathbb{Q})|\psi\rangle .
\end{aligned}
$$

Finally, by using $\mathbb{P}^{2}=\mathbb{P}$ and $\mathbb{Q}^{2}=\mathbb{Q}$, one obtains the system

$$
i \partial_{t}\left(\begin{array}{c}
\left|\psi_{p}\right\rangle \\
\left|\psi_{q}\right\rangle
\end{array}\right)=\left(\begin{array}{cc}
H_{p} & V \\
V^{\dagger} & H_{q}
\end{array}\right)\left(\begin{array}{l}
\left|\psi_{p}\right\rangle \\
\left|\psi_{q}\right\rangle
\end{array}\right),
$$

where

$$
\begin{gathered}
H_{p}=\mathbb{P} H \mathbb{P}, \\
H_{q}=\mathbb{Q} H \mathbb{Q}, \\
V=\mathbb{P} H \mathbb{Q}, \\
\left|\psi_{P}\right\rangle=\mathbb{P}|\psi\rangle, \\
\left|\psi_{Q}\right\rangle=\mathbb{Q}|\psi\rangle .
\end{gathered}
$$

Here $\left|\psi_{p}\right\rangle$ and $\left|\psi_{q}\right\rangle$ are respectively the projection of the state $|\psi\rangle$ in the relevant and irrelevant subspaces. In the interaction picture,

$$
\begin{aligned}
& \left|\psi_{p}\right\rangle=e^{-i H_{p} t}\left|\hat{\phi}_{p}\right\rangle, \\
& \left|\psi_{q}\right\rangle=e^{-i H_{q} t}\left|\hat{\phi}_{q}\right\rangle,
\end{aligned}
$$

the free evolution is eliminated:

$$
\begin{gathered}
i \partial_{t}\left|\hat{\phi}_{p}\right\rangle=e^{+i H_{p} t} V e^{-i H_{q} t}\left|\hat{\phi}_{q}\right\rangle \equiv \hat{V}(t)\left|\hat{\phi}_{q}\right\rangle, \\
i \partial_{t}\left|\hat{\phi}_{q}\right\rangle=e^{+i H_{q} t} V^{\dagger} e^{-i H_{p} t}\left|\hat{\phi}_{p}\right\rangle \equiv \hat{V}^{\dagger}(t)\left|\hat{\phi}_{p}\right\rangle .
\end{gathered}
$$

We introduce the operators $U_{p}$ and $U_{q}$ that diagonalize $H_{p}$ and $H_{q}$ :

$$
\begin{aligned}
& H_{p}=U_{p} \lambda_{p} U_{p}^{\dagger}, \\
& H_{q}=U_{q} \lambda_{q} U_{q}^{\dagger},
\end{aligned}
$$

where $\lambda_{p}=\operatorname{diag}\left\{\lambda_{p}^{i}\right\}$ and $\lambda_{q}=\operatorname{diag}\left\{\lambda_{q}^{i}\right\}$, and defining

$$
\begin{aligned}
\left|\tilde{\phi}_{p}\right\rangle & =U_{p}^{\dagger}\left|\hat{\phi}_{p}\right\rangle, \\
\left|\tilde{\phi}_{q}\right\rangle & =U_{q}^{\dagger}\left|\hat{\phi}_{q}\right\rangle,
\end{aligned}
$$

we have

$$
\begin{aligned}
i \partial_{t}\left|\tilde{\phi}_{p}\right\rangle & =\tilde{V}(t)\left|\tilde{\phi}_{q}\right\rangle, \\
i \partial_{t}\left|\tilde{\phi}_{q}\right\rangle & =\tilde{V}^{\dagger}(t)\left|\tilde{\phi}_{p}\right\rangle,
\end{aligned}
$$

and

$$
\tilde{V}(t)=U_{p}^{\dagger} \hat{V}(t) U_{q}=e^{i \lambda_{p} t} \hat{V}(t) e^{-i \lambda_{q} t} .
$$

Assuming that the population of the irrelevant space is initially zero $\left|\hat{\phi}_{q}(0)\right\rangle=|0\rangle$, the formal solution of system (A4) is, in components,

$$
\tilde{\phi}_{q, k}(t)=-i \sum_{j} \int_{0}^{t} d t^{\prime} V_{j k}^{*} e^{i\left(\lambda_{q, k}-\lambda_{p, j}\right) t^{\prime}} \tilde{\phi}_{p, j}\left(t^{\prime}\right) .
$$

After partial integration one finds

$$
\begin{aligned}
\tilde{\phi}_{q, k}(t)= & -i \sum_{j}\left\{\left.\frac{e^{i\left(\lambda_{q, k}-\lambda_{p . j}\right) t^{\prime}}}{i\left(\lambda_{q, k}-\lambda_{p . j}\right)} V_{j k}^{*} \tilde{\phi}_{p, j}\left(t^{\prime}\right)\right|_{0} ^{t}\right. \\
& \left.-\int_{0}^{t} d t^{\prime} \frac{e^{i\left(\lambda_{q, k}-\lambda_{p . j}\right) t^{\prime}}}{i\left(\lambda_{q, k}-\lambda_{p . j}\right)} \frac{d}{d t^{\prime}} \tilde{\phi}_{p, j}\left(t^{\prime}\right)\right\} .
\end{aligned}
$$

The second integral can be neglected because, by carrying on the partial integration procedure, the next term is of the order of $\left(\lambda_{q, k}-\lambda_{p . j}\right)^{-2}$. Indeed, for a large spectral separation between the relevant and the irrelevant subspaces, $\left|\lambda_{Q}^{k}-\lambda_{P}^{j}\right| \gg 1$, and when the edge term is zero one has

$$
\tilde{\phi}_{q, k}(t)=-\sum_{j} \tilde{W}_{k j}(t) \tilde{\phi}_{p, j}(t),
$$

where

$$
\tilde{W}_{k j}(t)=V_{j k}^{*} \frac{\exp \left[i\left(\lambda_{Q}^{k}-\lambda_{P}^{j}\right) t\right]}{\lambda_{Q}^{k}-\lambda_{P}^{j}} .
$$

Finally one find that the effective Schrödinger equation for the relevant space dynamics is

$$
i \partial_{t}\left|\psi_{p}\right\rangle \simeq H_{\mathrm{eff}}\left|\psi_{p}\right\rangle
$$

where

$$
H_{\mathrm{eff}}=H_{p}-V W
$$

and $W$ satisfies

$$
H_{q} W-W H_{p}=V^{\dagger} .
$$


When $H_{p}$ consists of a degenerate levels with energy $E_{0}$ the above effective theory corresponds to the usual degenerate perturbation theory [73]:

$$
\left\langle\phi\left|H_{\mathrm{eff}}\right| \phi^{\prime}\right\rangle \simeq\left\langle\phi|H| \phi^{\prime}\right\rangle+\sum_{m \in \mathcal{H}_{q}} \frac{\langle\phi|V| m\rangle\left\langle m|V| \phi^{\prime}\right\rangle}{E_{0}-E_{m}}+\cdots,
$$

where $\mathcal{H}_{p}$ and $\mathcal{H}_{q}$ are the relevant and irrelevant Hilbert subspaces and $|\phi\rangle,\left|\phi^{\prime}\right\rangle \in \mathcal{H}_{p}$.

We derive explicitly the effective Hamiltonian for a BoseHubbard model (1). The Hilbert space $\mathcal{H}$ with fixed number of particle $M$ in a chain with length $L$ has size

$$
\operatorname{dim} \mathcal{H}=\frac{(M+L-1) !}{M !(L-1) !} .
$$

The relevant space $\mathcal{H}_{p} \equiv \mathcal{H}_{M}$ is defined as

$$
\mathcal{H}_{p}=\left\{\left|\psi_{m}^{b}\right\rangle \in \mathcal{H}:\left|\psi_{m}^{b}\right\rangle=\left|0, \ldots, 0, M_{m}, 0, \ldots, 0\right\rangle\right\},
$$

where $M_{m}=M$. Clearly $\operatorname{dim} \mathcal{H}_{p}=L$. The irrelevant space $\mathcal{H}_{q}=\mathcal{H} \backslash \mathcal{H}_{p}$ is

$$
\begin{array}{r}
\mathcal{H}_{q}=\left\{\left|\psi_{m}^{u}\right\rangle \in \mathcal{H}:\left|\psi_{m}^{u}\right\rangle=\left|n_{1}, \ldots, n_{L}\right\rangle,\right. \\
\text { where } \left.\sum_{j} n_{j}=M \text { and } n_{j} \neq M\right\} .
\end{array}
$$

Given the above definitions we rename the basis of the Hilbert space as $|m\rangle, m=1, \ldots, \operatorname{dim} \mathcal{H}$ such that $|m\rangle=\left|\psi_{m}^{b}\right\rangle$ for $m=1, \ldots, L$. The Hamiltonian then takes the following block form:

$$
H=\left(\begin{array}{c|c}
H_{p} & V \\
\hline V^{\dagger} & H_{q}
\end{array}\right),
$$

where each block can be evaluated explicitly with the following projection operators:

$$
\begin{gathered}
\mathbb{P}=\sum_{\substack{m=1 \\
\operatorname{dim} H_{q}}}^{L}|m\rangle\left\langle\psi_{m}^{b}\right|, \\
\mathbb{Q}=\sum_{m=1}|L+m\rangle\left\langle\psi_{m}^{u}\right| .
\end{gathered}
$$

Clearly, $\operatorname{dim} \mathcal{H}_{p}=(L, L), \operatorname{dim} \mathcal{H}_{q}=(\operatorname{dim} \mathcal{H}-L, \operatorname{dim} \mathcal{H}-L)$, and $\operatorname{dim} V=(L, \operatorname{dim} \mathcal{H}-L)$.

The effective Hamiltonian (A26) can be computed explicitly by using a series expansion for large $U_{j}=U$. The relevant Hamiltonian for the Bose-Hubbard model (1) takes the simple form

$$
H_{p}=\sum_{m=1}^{L} \frac{U}{2} M(M-1)|m\rangle\left\langle m\left|-\sum_{m=1}^{L} \mu_{m} M\right| m\right\rangle\langle m| .
$$

Similarly $H_{q}$ and $V$ can be computed explicitly. The effective model can be obtained by solving Eqs. (A26) and (A27). To find the $W$ matrix we vectorize (see Appendix C) Eq. (A27) as

$$
G \operatorname{vec}(W)=\operatorname{vec}\left(V^{\dagger}\right)
$$

where

$$
G=\left(\mathbf{1}_{\operatorname{dim} H_{p}} \otimes H_{q}\right)-\left(H_{p}^{t} \otimes \mathbf{1}_{\operatorname{dim} H_{q}}\right) .
$$

It it is convenient to write $G=G^{\text {large }}+G^{\text {small }}$ where

$$
\begin{aligned}
G^{\text {large }} & =\mathbf{1}_{\operatorname{dim} H_{p}} \otimes H_{q}^{\text {large }}-\left(H_{p}^{\text {large }}\right)^{t} \otimes \mathbf{1}_{\operatorname{dim} H_{q}}, \\
G^{\text {small }} & =\mathbf{1}_{\operatorname{dim} H_{p}} \otimes H_{q}^{\text {small }}-\left(H_{p}^{\text {small }}\right)^{t} \otimes \mathbf{1}_{\operatorname{dim} H_{p}},
\end{aligned}
$$

and $H^{\text {large }}$ is the part of the Hamiltonian (1) that contains the terms in $U: H^{\text {large }}=\sum_{j=1}^{L} \frac{U}{2} n_{j}\left(n_{j}-1\right)$ and $H^{\text {small }}=H-$ $H^{\text {large }}$. The system (A36) can be formally solved by taking the inverse of the $G$ matrix as

$$
\operatorname{vec}(W)=\frac{1}{G^{\text {large }}+G^{\text {small }}} \operatorname{vec}\left(V^{\dagger}\right)
$$

and using the following identity, valid for two operators $A$ and $B$ :

$$
\frac{1}{A+B}=\frac{1}{A}\left(1-B \frac{1}{A+B}\right) .
$$

Indeed one can easily find that

$$
\begin{aligned}
\frac{1}{A}\left(1-B \frac{1}{A+B}\right)= & A^{-1}\left[1-B(A+B)^{-1}\right] \\
= & A^{-1}\left[(A+B)(A+B)^{-1}\right. \\
& \left.-B(A+B)^{-1}\right] \\
= & A^{-1}\left[A(A+B)^{-1}\right]=\frac{1}{A+B} .
\end{aligned}
$$

By using recursively Eq. (A41) one finds the Dyson expansion

$$
\frac{1}{G^{\text {large }}+G^{\text {small }}}=\left(G^{\text {large }}\right)^{-1} \sum_{n=0}^{+\infty}(-1)^{n}\left[G^{\text {small }}\left(G^{\text {large }}\right)^{-1}\right]^{n},
$$

which corresponds to a series expansion in the onsite interaction parameter $U$ (which is contained in $G^{\text {large }}$ ). Moreover,

$$
G^{\text {large }}=-\frac{U}{2} \sum_{m=1}^{L} \sum_{n=1}^{\operatorname{dim} H_{q}} g(n, m)|L+n, m\rangle\langle L+n, m|,
$$

where

$$
g(n, m)=\sum_{j} M(M-1) \delta_{j m}-n_{j}\left(n_{j}-1\right)>0,
$$

since in $\mathcal{H}_{q}$ it is $n_{j}<M$ and $\sum_{j} n_{j}=M$. Therefore, $G^{\text {large }}$ is diagonal and nonsingular for each value of $M$. By truncating the expansion (A43) at the relevant order $n$ in $U$ one obtains the matrix $W$ from Eq. (A40) and then the effective Hamiltonian from Eq. (A26), valid to the $n$th order.

As an example of the general procedure outlined above we consider explicitly the first-order solution where $M=2$ and 
(A43) reduces to

$$
\begin{gathered}
\left(G^{\text {large }}+G^{\text {small }}\right)^{-1} \approx\left(G^{\text {large }}\right)^{-1} \\
=-U^{-1} \sum_{m=1}^{L} \sum_{n=1}^{\operatorname{dim} H_{q}}|L+n, m\rangle\langle L+n, m| \\
=-\frac{\mathbb{1}_{q} \otimes \mathbb{1}_{p}}{U} .
\end{gathered}
$$

Therefore, according to (A36), $W=-V^{\dagger} / U$, so

$$
\begin{aligned}
H_{\mathrm{eff}} \simeq & -\sum_{m=1}^{L} \mu_{m} M|m\rangle\langle m| \\
& +\sum_{m, m^{\prime}=1}^{L}|m\rangle \frac{\left\langle\psi_{m}^{b}\left|V \mathbb{Q} V^{\dagger}\right| \psi_{m^{\prime}}^{b}\right\rangle}{U}\left\langle m^{\prime}\right|,
\end{aligned}
$$

where we have explicitly omitted the terms proportional to the identity. For the interaction term $V$ between $\mathcal{H}_{p}$ and $\mathcal{H}_{q}$ we observe that the only nonzero matrix elements are $\left\langle\psi_{m}^{b}\left|a_{j} a_{j+1}^{\dagger}\right| \psi_{n}^{u}\right\rangle$ (as well as their Hermitian conjugate) when

$$
\begin{gathered}
\left|\psi_{m}^{b}\right\rangle=\left|0, \ldots, M_{j}, \ldots, 0\right\rangle, \\
\left|\psi_{n}^{u}\right\rangle=\left|0, \ldots, 1_{j},(M-1)_{j+1}, \ldots, 0\right\rangle .
\end{gathered}
$$

These can give rise to a hopping from $|m\rangle$ to $|m+1\rangle$ only for $M=2$. Indeed, this is done with the following steps: Starting from $|m\rangle=\left|0, \ldots, 2_{m}, 0, \ldots\right\rangle$ the operator $a_{m} a_{m+1}^{\dagger}$ in $V$ maps this state to $\left|0, \ldots, 1_{m}, 1_{m+1}, 0, \ldots\right\rangle$ which is in $\mathcal{H}_{q}$. Then the operator $a_{m} a_{m+1}^{\dagger}$ in $V^{\dagger}$ maps that state to $|m+1\rangle=\left|0, \ldots, 2_{m+1}, 0, \ldots\right\rangle$. By generalizing the above argument, with simple calculations one finds then

$$
H_{\mathrm{eff}}^{M=2} \simeq-\sum_{m=1}^{L} \mu_{m} M|m\rangle\langle m|+\sum_{m}^{L-1} \frac{J_{m}^{2}}{2 U}(|m\rangle\langle m+1|+\text { H.c. }) \text {. }
$$

The generalization to higher values of $M$ proceeds along the same lines. For instance, for $M=3$ one has to consider the second-order expansion in (A43) which depends also on $G^{\text {small }}$. Indeed, an effective hopping can happen only via a three step procedure

$$
\begin{aligned}
|m\rangle & =\left|0, \ldots, 3_{m}, 0, \ldots\right\rangle \rightarrow\left|0, \ldots, 2_{m}, 1_{m+1} 0, \ldots\right\rangle \\
& \rightarrow\left|0, \ldots, 1_{m}, 2_{m+1} 0, \ldots\right\rangle \\
& \rightarrow\left|0, \ldots, 3_{m+1} 0, \ldots\right\rangle \equiv|m+1\rangle .
\end{aligned}
$$

By doing explicit calculations we find the effective Hamiltonians mentioned in the main text.

\section{APPENDIX B: MINIMAL ENGINEERING OF THE THREE-PARTICLE BOUND-STATE PROPAGATION}

The state transfer fidelity of the three-particle bound state can be improved by introducing an optimal coupling scheme; namely, tuning the first and the last tunneling coupling to $J_{1}=J_{L-1}=J_{0}$ and the rest of the chain to $J_{j}=J$. We find that, in order to delocalize the bound state, two pairs of localized fields in the endpoints are necessary, respectively $\mu_{j}=-\beta_{1}\left(\delta_{j, 1}+\delta_{j, L}\right)$ and $\mu_{j}=-\beta_{2}\left(\delta_{j, 2}+\delta_{j, L-1}\right)$ where $\beta_{1}=\left(2 J^{2}-J_{0}^{2}\right) / 8 U$ and $\beta_{2}=\left(J^{2}-J_{0}^{2}\right) / 8 U$. In this case the beam-splitting condition for $L \gg 1$ is realized when a local field $\mu_{j}=-\beta \delta_{j, L / 2+1}$ with strength $\beta=\tilde{\beta} J^{3} / 8 U^{2}$ is added in the middle of the chain. The effective Hamiltonian is it in this case

$$
\begin{aligned}
& H_{\mathrm{opt}}^{I I I} / 2 J_{\mathrm{eff}}^{I I I}
\end{aligned}
$$

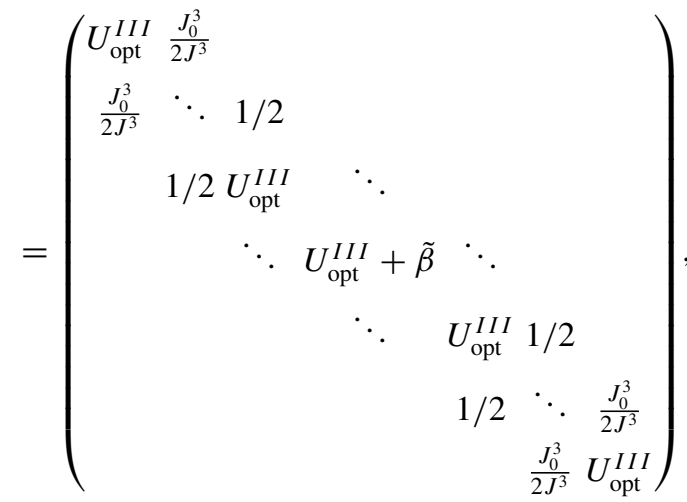

where $J_{\text {eff }}^{I I I}=3 J^{3} / 16 U^{2}$, and $U_{\text {opt }}^{I I I}=8(U / J)^{3}+2 U / J+$ $16(J / U)^{2}$. To have a perfectly balanced beam splitter in this single-particle Hamiltonian, as shown in Ref. [37], one needs $\tilde{\beta}=1$. Therefore, $\beta^{50-50}=J^{3} / 8 U^{2}$. Our method is straightforwardly generalizable to bound states with a higher number of particles.

\section{APPENDIX C: NUMERICAL SOLUTION OF THE MASTER EQUATION}

To solve the master equation (8) we exploit a vectorization procedure [74] which consists of representing a matrix as a vector, by using its representation in the canonical basis with a column ordering. For instance, for a generic $2 \times 2$ matrix $A$, its vectorization $\operatorname{vec}(A)$ is

$$
\begin{aligned}
& A=\left(\begin{array}{ll}
A_{11} & A_{12} \\
A_{21} & A_{22}
\end{array}\right)=A_{11}\left(\begin{array}{ll}
1 & 0 \\
0 & 0
\end{array}\right) \\
& +A_{21}\left(\begin{array}{ll}
0 & 0 \\
1 & 0
\end{array}\right)+\cdots, \\
& \operatorname{vec}(A)=\left(\begin{array}{llll}
A_{11}, & A_{21}, & A_{12}, & A_{22}
\end{array}\right)^{t} .
\end{aligned}
$$

For a general size matrix $\rho$ the latter procedure corresponds to the mapping $v_{(k-1) L+j}=\rho_{j k}$ where $v=\operatorname{vec}(\rho)$. Once chosen this base the action of an operator $H$ on the left or the right of the density matrix $\rho$ can be written as

$$
\begin{aligned}
& H \rho=\left(\mathbf{1}_{L} \otimes H\right) \operatorname{vec}(\rho), \\
& \rho H=\left(H^{t} \otimes \mathbf{1}_{L}\right) \operatorname{vec}(\rho),
\end{aligned}
$$

and for the dissipative part, by using the identities

$$
\begin{aligned}
\operatorname{vec}(A B C) & =\left(C^{t} \otimes A\right) \operatorname{vec}(B)=\left(\mathbf{1}_{L} \otimes A B\right) \operatorname{vec}(C) \\
& =\left(C^{t} B^{t} \otimes \mathbf{1}_{L}\right) \operatorname{vec}(A), \\
\operatorname{vec}(A B) & =\left(\mathbf{1}_{L} \otimes A\right) \operatorname{vec}(B)=\left(B^{t} \otimes \mathbf{1}_{L}\right) \operatorname{vec}(A),
\end{aligned}
$$


we find that

$$
\begin{gathered}
n_{i} n_{i} \rho=\left(\mathbf{1}_{L} \otimes n_{i} n_{i}\right) \operatorname{vec}(\rho), \\
\rho n_{i} n_{i}=\left[\left(n_{i} n_{i}\right)^{t} \otimes \mathbf{1}_{L}\right] \operatorname{vec}(\rho), \\
n_{i} \rho n_{i}=\left[\left(n_{i}\right)^{t} \otimes n_{i}\right] \operatorname{vec}(\rho),
\end{gathered}
$$

hence if $H$ and $\rho$ describe a fixed particle number subspace one obtains the vectorized version of the master equation (8); namely,

$$
\operatorname{vec}(\dot{\rho})=\mathcal{L}_{v} \operatorname{vec}(\rho)
$$

where the operator $\mathcal{L}_{v}$ is defined as

$$
\begin{aligned}
\mathcal{L}_{v}= & -i\left\{\left(\mathbf{1}_{L} \otimes H\right)-\left(H^{t} \otimes \mathbf{1}_{L}\right)\right\}-\gamma \sum_{j}\left\{\left(n_{j}^{t} \otimes n_{j}\right)\right. \\
& \left.-\frac{1}{2}\left(\mathbf{1}_{L} \otimes n_{j}^{2}\right)-\frac{1}{2}\left[\left(n_{j}^{2}\right)^{t} \otimes \mathbf{1}_{L}\right]\right\} .
\end{aligned}
$$

[1] C. Weitenberg, M. Endres, J. F. Sherson, M. Cheneau, P. Schauß, T. Fukuhara, I. Bloch, and S. Kuhr, Nature (London) 471, 319 (2011).

[2] P. M. Preiss, R. Ma, E. Tai, A. Lukin, M. Rispoli, P. Zupancic, Y. Lahini, R. Islam, and M. Greiner, Science 347, 1229 (2015).

[3] T. Fukuhara, P. Shauß, M. Endres, S. Hild, M. Cheneau, I. Bloch, and C. Gross, Nature (London) 502, 76 (2013).

[4] T. Fukuhara et al., Nat. Phys. 9, 235 (2013).

[5] S. Murmann, A. Bergschneider, V. M. Klinkhamer, G. Zürn, T. Lompe, and S. Jochim, Phys. Rev. Lett. 114, 080402 (2015).

[6] P. M. Preiss, R. Ma, M. E. Tai, J. Simon, and M. Greiner, Phys. Rev. A 91, 041602 (2015).

[7] C. Robens, S. Brakhane, D. Meschede, and A. Alberti, in Proceedings of the XXII International Conference (Laser Spectroscopy, Singapore, 2015), pp. 1-15.

[8] A. Celi, P. Massignan, J. Ruseckas, N. Goldman, I. B. Spielman, G. Juzeliūnas and M. Lewenstein, Phys. Rev. Lett. 112, 043001 (2014).

[9] M. Mancini et al., Science 349, 1510 (2015).

[10] G. De Chiara and A. Sanpera, J. Low Temp. Phys. 165, 292 (2011).

[11] R. Islam, R. Ma, P. M. Preiss, T. E. Tai, A. Lukin, M. Rispoli, and M. Greiner, Nature (London) 528, 77 (2015).

[12] P. Zupancic, P. M. Preiss, R. Ma, A. Lukin, M. E. Tai, M. Rispoli, R. Islam, and M. Greiner, Opt. Express 24, 13881 (2016).

[13] H. Ott, Rep. Prog. Phys. 79, 054401 (2016).

[14] D. K. Hoffmann, B. Deissler, W. Limmer, and J. H. Denschlag, Appl. Phys. B: Lasers Opt. 122, 227 (2016).

[15] E. Rocco, R. N. Palmer, T. Valenzuela, V. Boyer, A. Freise, and K. Bongs, New J. Phys. 16, 093046 (2014).

[16] T. Kovachy, P. Asenbaum, C. Overstreet, C. A. Donnelly, S. M. Dickerson, A. Sugarbaker, J. M. Hogan, and M. A. Kasevich, Nature (London) 528, 530 (2015).

[17] H. Lee, P. Kok, and J. P. Dowling, J. Mod. Opt. 49, 2325 (2002).

[18] J. P. Dowling, Contemp. Phys. 49, 125 (2008).

[19] R. Demkowicz-Dobrzański, M. Jarzyna, and J. Kołodyński, in Progress in Optics, edited by E. Wolf (North-Holland Pub. Co., Amsterdam, 2015), Vol. 60.

[20] C. Gross, T. Zibold, E. Nicklas, J. Estève, and M. K. Oberthaler, Nature (London) 464, 1165 (2010).

[21] C. Gross, J. Phys. B: At., Mol. Opt. Phys. 45, 103001 (2012).

[22] Y. Israel, S. Rosen, and Y. Silberberg, Phys. Rev. Lett. 112, 103604 (2014).

[23] H. F. Hofmann and T. Ono, Phys. Rev. A 76, 031806 (2007).
[24] I. Afek, O. Ambar, and Y. Silberberg, Science 328, 879 (2010).

[25] Y. Israel, I. Afek, S. Rosen, O. Ambar, and Y. Silberberg, Phys. Rev. A 85, 022115 (2012).

[26] P. Kok, H. Lee, and J. P. Dowling, Phys. Rev. A 65, 052104 (2002).

[27] W. J. Mullin and F. Laloë, J. Low Temp. Phys. 162, 250 (2010).

[28] K. Stiebler, B. Gertjerenken, N. Teichmann, and C. Weiss, J. Phys. B: At., Mol. Opt. Phys. 44, 055301 (2011).

[29] G. Kordas, S. Wimberger, and D. Witthaut, Europhys. Lett. 100, 30007 (2012).

[30] A. M. Leung, K. W. Mahmud, and W. P. Reinhardt, Mol. Phys. 110, 801 (2012).

[31] L. Dell'Anna, G. Mazzarella, V. Penna, and L. Salasnich, Phys. Rev. A 87, 053620 (2013).

[32] K. Winkler, G. Thalhammer, F. Lang, R. Grimm, J. Hecker Denschlag, A. J. Daley, A. Kantian, H. P. Büchler, and P. Zoller, Nature (London) 76, 033606 (2007).

[33] D. Petrosyan, B. Schmidt, J. R. Anglin, and M. Fleischhauer, Phys. Rev. A 76, 033606 (2007).

[34] S. Fölling, S. Trotzky, P. Cheinet, M. Feld, R. Saers, A. Widera, T. Müller, and I. Bloch, Nature (London) 448, 1029 (2007).

[35] M. Valiente and D. Petrosyan, J. Phys. B: At., Mol. Opt. Phys. 41, 161002 (2008).

[36] M. Valiente, D. Petrosyan, and A. Saenz, Phys. Rev. A 81, 011601 (2010).

[37] E. Compagno, L. Banchi, and S. Bose, Phys. Rev. A 92, 022701 (2015).

[38] L. Banchi, E. Compagno, and S. Bose, Phys. Rev. A 91, 052323 (2015).

[39] R. A. Pinto, M. Haque, and S. Flach, Phys. Rev. A 79, 052118 (2009).

[40] M. Haque, Phys. Rev. A 82, 012108 (2010).

[41] M. Bello, C. E. Creffield, and G. Platero, Sci. Rep. 6, 22562 (2016).

[42] M. Bello, C. E. Creffield, and G. Platero, arXiv:1608.00162.

[43] A. R. Kolovsky, J. Link, and S. Winberger, New J. Phys. 14, 075002 (2012).

[44] D. N. Maksimov and A. R. Kolovsky, Phys. Rev. A 89, 063612 (2014).

[45] N. Jia, L. Banchi, A. Bayat, G. Dong, and S. Bose, Sci. Rep. 5, 13665 (2015).

[46] V. Alba, K. Saha, and M. Haque, J. Stat. Mech.: Theory Exp. (2013) P10018. 
[47] M. Lewenstein, A. Sanpera, and V. Ahufinger, Ultracold Atoms in Optical Lattices (Oxford University Press, Oxford, 2012), p. 51.

[48] Y. Lahini, M. Verbin, S. D. Huber, Y. Bromberg, R. Pugatch, and Y. Silberberg, Phys. Rev. A 86, 011603 (2012).

[49] X. Qin, Y. Ke, X. Guan, Z. Li, N. Andrei, and C. Lee, Phys. Rev. A 90, 062301 (2014).

[50] A. G. Volosniev, D. Petrosyan, M. Valiente, D. V. Fedorov, A. S. Jensen, and N. T. Zinner, Phys. Rev. A 91, 023620 (2015).

[51] S. Lorenzo, T. J. G. Apollaro, S. Paganelli, G. M. Palma, and F. Plastina, Phys. Rev. A 91, 042321 (2015).

[52] T. J. G. Apollaro, S. Lorenzo, A. Sindona, S. Paganelli, G. L. Giorgi and F. Plastina, Phys. Scr. T165, 014036 (2015).

[53] R. Sousa and Y. Omar, New J. Phys. 16, 123003 (2014).

[54] S. Paganelli, S. Lorenzo, T. J. G. Apollaro, F. Plastina, and G. L. Giorgi, Phys. Rev. A 87, 062309 (2013).

[55] M. Christandl, N. Datta, A. Ekert, and A. J. Landahl, Phys. Rev. Lett. 92, 187902 (2004).

[56] V. Kostak, G. M. Nikolopoulos, and I. Jex, Phys. Rev. A 75, 042319 (2007).

[57] A. Kay, Int. J. Quantum Inform. 08, 641 (2010).

[58] V. X. Genest, L. Vinet, and A. Zhedanov, Ann. Phys. (NY) 371, 348 (2016).

[59] J. M. Lemay, L. Vinet, and A. Zhedanov, J. Phys. A: Math. Theor. 49, 335302 (2016).

[60] L. Banchi and R. Vaia, J. Math. Phys. 54, 043501 (2013).

[61] L. Banchi, T. J. G. Apollaro, A. Cuccoli, R. Vaia, and P. Verrucchi, New J. Phys. 13, 123006 (2011).
[62] T. J. G. Apollaro, L. Banchi, A. Cuccoli, R. Vaia, and P. Verrucchi, Phys. Rev. A 85, 052319 (2012).

[63] A. Zwick, G. A. Álvarez, J. Stolze, and O. Osenda, Phys. Rev. A 84, 022311 (2011).

[64] A. K. Pavlis, G. M. Nikolopoulos, and P. Lambropoulos, Quantum Inf. Process. 15, 2553 (2016).

[65] G. Kordas, D. Witthaut, and S. Wimberger, Ann. Phys. (Berlin, Ger.) 527, 619 (2015)

[66] H. Pichler, A. J. Daley, and P. Zoller, Phys. Rev. A 82, 063605 (2010).

[67] S. Sarkar, S. Langer, J. Schachenmayer, and A. J. Daley, Phys. Rev. A 90, 023618 (2014).

[68] F. Laloë and W. J. Mullin, Found. Phys. 42, 53 (2011).

[69] P. Hauke, M. Heyl, L. Tagliacozzo, and P. Zoller, Nat. Phys. 12, 778 (2016).

[70] G. Mazzarella, L. Salasnich, A. Parola, and F. Toigo, Phys. Rev. A 83, 053607 (2011).

[71] J. Tangpanitanon, V. M. Bastidas, S. Al-Assam, P. Roushan, D. Jaksch, and D. G. Angelakis, Phys. Rev. Lett. 117, 213603 (2016).

[72] G. Watanabe, Phys. Rev. A 81, 021604 (2010).

[73] F. Mila and K. P. Schmidt, in Introduction to Frustrated Magnetism: Materials, Experiments, Theory, Chapter 20, Springer Series in Solid-State Sciences, Vol. 164, edited by C. Lacroix, F. Mila, and P. Mendels (Springer, Berlin, Heidelberg, 2011), pp. 537-559.

[74] M. Am-Shallem, A. Levy, I. Schaefer, and R. Kosloff, arXiv:1510.08634. 